\title{
Second-order asymptotics for quantum hypothesis testing in settings beyond i.i.d. - quantum lattice systems and more
}

\author{
Nilanjana Datta ${ }^{\mathrm{a}}$, Yan Pautrat ${ }^{\mathrm{b}}$, and Cambyse Rouzéa

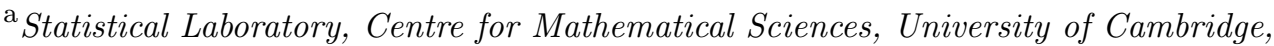 \\ Cambridge CB30WB, UK \\ ${ }^{\mathrm{b}}$ Laboratoire de Mathématiques d'Orsay, Univ. Paris-Sud, CNRS, Université Paris-Saclay, \\ 91405 Orsay, France
}

August 31, 2021

\begin{abstract}
Quantum Stein's Lemma is a cornerstone of quantum statistics and concerns the problem of correctly identifying a quantum state, given the knowledge that it is one of two specific states ( $\rho$ or $\sigma$ ). It was originally derived in the asymptotic i.i.d. setting, in which arbitrarily many (say, $n$ ) identical copies of the state $\left(\rho^{\otimes n}\right.$ or $\left.\sigma^{\otimes n}\right)$ are considered to be available. In this setting, the lemma states that, for any given upper bound on the probability $\alpha_{n}$ of erroneously inferring the state to be $\sigma$, the probability $\beta_{n}$ of erroneously inferring the state to be $\rho$ decays exponentially in $n$, with the rate of decay converging to the relative entropy of the two states. The second order asymptotics for quantum hypothesis testing, which establishes the speed of convergence of this rate of decay to its limiting value, was derived in the i.i.d. setting independently by Tomamichel and Hayashi, and Li. We extend this result to settings beyond i.i.d.. Examples of these include Gibbs states of quantum spin systems (with finite-range, translation-invariant interactions) at high temperatures, and quasi-free states of fermionic lattice gases.
\end{abstract}

\section{Introduction}

\section{Quantum Hypothesis Testing}

Quantum hypothesis testing concerns the problem of discriminating between two different quantum states ${ }^{1}$. It is fundamentally different from its classical counterpart, in which one discriminates between two different probability distributions, the difference arising essentially from the non-commutativity of quantum states of physical systems, which are at the heart of quantum information. Hence, discriminating between different states of a quantummechanical system is of paramount importance in quantum information-processing tasks. In the language of hypothesis testing, one considers two hypotheses - the null hypothesis $H_{0}: \rho$

\footnotetext{
${ }^{1}$ It is often referred to as binary quantum hypothesis testing, to distinguish it from the case in which there are more than two states.
} 
and the alternative hypothesis $H_{1}: \sigma$, where $\rho$ and $\sigma$ are two quantum states. In an operational setting, say Bob receives a state $\omega$ with the knowledge that either $\omega=\rho$ or $\omega=\sigma$. His goal is then to infer which hypothesis is true, i.e., which state he has been given, by means of a measurement on the state he receives. The measurement is given most generally by a $\operatorname{POVM}\{T, \mathbb{I}-T\}$ where $0 \leq T \leq \mathbb{I}$. Adopting the nomenclature from classical hypothesis testing, we refer to $T$ as a test. The probability that Bob correctly guesses the state to be $\rho$ is then equal to $\operatorname{Tr}(T \rho)$, whereas his probability of correctly guessing the state to be $\sigma$ is $\operatorname{Tr}((\mathbb{I}-T) \sigma)$. Bob can erroneously infer the state to be $\sigma$ when it is actually $\rho$ or vice versa. The corresponding error probabilities are referred to as the type I error and type II error respectively. They are given as follows:

$$
\alpha(T):=\operatorname{Tr}((\mathbb{I}-T) \rho), \quad \beta(T):=\operatorname{Tr}(T \sigma),
$$

where $\alpha(T)$ is the probability of accepting $H_{1}$ when $H_{0}$ is true, while $\beta(T)$ is the probability of accepting $H_{0}$ when $H_{1}$ is true. Obviously, there is a trade-off between the two error probabilities, and there are various ways to jointly optimize them, depending on whether or not the two types of errors are treated on an equal footing. In the setting of symmetric hypothesis testing, one minimizes the total probability of error $\alpha(T)+\beta(T)$, whereas in asymmetric hypothesis testing one minimizes the type II error under a suitable constraint on the type I error.

Quantum hypothesis testing was originally studied in the asymptotic i.i.d. setting in which Bob is provided not with just a single copy of the state but with multiple (say $n$ ) identical copies of the state, and he is allowed to do a joint measurement on all these copies. The optimal asymptotic performance in the different settings is quantified by the following exponential decay rates, evaluated in the asymptotic limit $(n \rightarrow \infty)$ : $(i)$ the optimal exponential decay rate of the sum of type I and type II errors, $(i i)$ the optimal exponential decay rate of the type II error under the assumption that the type I error decays with a given exponential speed, and (iii) the optimal exponential decay rate of the type II error under the assumption that the type I error remains bounded. The first of these corresponds to the symmetric setting while the other two to the asymmetric setting; $(i)$ is given by the quantum Chernoff bound [4, 34, (ii) is given by the Hoeffding bound [14, 32, 35] while (iii) is given by quantum Stein's lemma [21, 36. In this paper we will restrict attention to the asymmetric setting of quantum Stein's Lemma and its refinement, and hence we elaborate on it below.

\section{Quantum Stein's lemma and its refinement: asymptotic i.i.d. setting}

Suppose that the state $\omega_{n}$ that Bob receives is either the state $\rho_{n}:=\rho^{\otimes n}$ or the state $\sigma_{n}:=\sigma^{\otimes n}$, with $\rho$ and $\sigma$ being states on a finite-dimensional Hilbert space $\mathcal{H}$. In the setting of quantum Stein's lemma, the quantity of interest is the optimal asymptotic type II error exponent, which is given, for any $\varepsilon \in(0,1)$, by

$$
\lim _{n \rightarrow \infty}-\frac{1}{n} \log \beta_{n}(\varepsilon), \quad \text { where } \beta_{n}(\varepsilon):=\inf _{0 \leq T_{n} \leq \mathbb{I}_{n}}\left\{\beta\left(T_{n}\right) \mid \alpha\left(T_{n}\right) \leq \varepsilon\right\} .
$$

Here $\alpha\left(T_{n}\right)=\operatorname{Tr}\left[\left(\mathbb{I}_{n}-T_{n}\right) \rho_{n}\right]$ and $\beta\left(T_{n}\right)=\operatorname{Tr}\left[T_{n} \sigma_{n}\right]$, with $\mathbb{I}_{n}$ being the identity operator acting on $\mathcal{H}^{\otimes n}$. Quantum Stein's lemma (see [21, 36]) establishes that

$$
\lim _{n \rightarrow \infty}-\frac{1}{n} \log \beta_{n}(\varepsilon)=D(\rho \| \sigma) \quad \forall \varepsilon \in(0,1),
$$


where $D(\rho \| \sigma)$ denotes the quantum relative entropy defined in Equation (2.4). Moreover, it states that the minimal asymptotic type I error jumps discontinuously from 0 to 1 as the asymptotic type II error exponent crosses the value $D(\rho \| \sigma)$ from below: see Figure 1, which is a plot of the function

$$
\alpha_{\infty}^{(1)}\left(t_{1}\right)=\inf \left\{\limsup _{n} \alpha\left(T_{n}\right) \mid-\liminf _{n} \frac{1}{n} \log \beta\left(T_{n}\right) \geq t_{1}\right\} .
$$

However, this discontinuous dependence of the minimal asymptotic type I error on the asymptotic type II error exponent is a manifestation of the coarse-grained analysis underlying the Quantum Stein's lemma, in which only the linear term (in $n$ ) of the type II error exponent $\left(-\log \beta\left(T_{n}\right)\right)$ is considered.

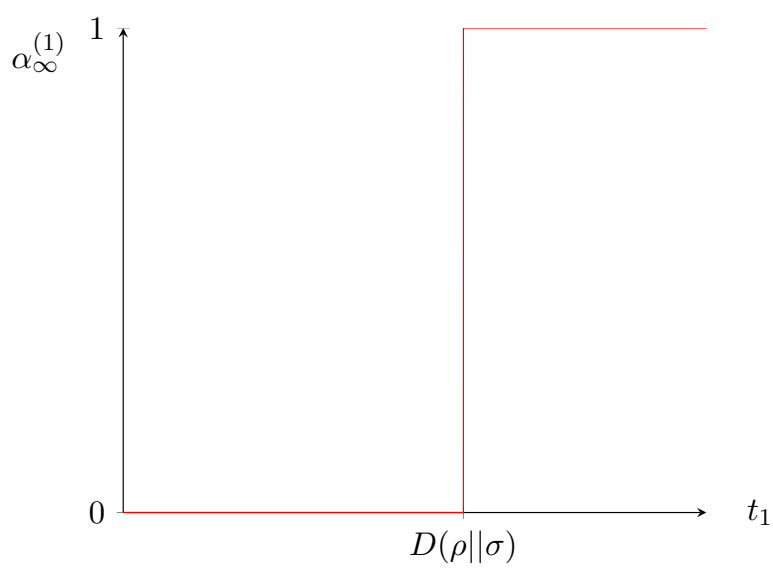

Figure 1: The minimal asymptotic type I error $\alpha_{\infty}^{(1)}\left(t_{1}\right)$, defined in Equation 1.2 .

More recently, Li 28] and Tomamichel and Hayashi 44] independently showed that this discontinuity vanishes under a more refined analysis of the type II error exponent, in which its second order (i.e. order $\sqrt{n}$ ) term is retained, in addition to the linear term. This analysis is referred to as the second order asymptotics for (asymmetric) quantum hypothesis testing, since it involves the evaluation of $\left(-\log \beta_{n}(\varepsilon)\right)$ up to second order. It was proved in [44, 28] to be given by:

$$
-\log \beta_{n}(\varepsilon)=n D(\rho \| \sigma)+\sqrt{n V(\rho \| \sigma)} \Phi^{-1}(\varepsilon)+\mathcal{O}(\log n),
$$

where $\Phi$ denotes the cumulative distribution function (c.d.f.) of a standard normal distribution, and $V(\rho \| \sigma)$ is called the quantum information variance and is defined in Equation (2.5). The above expansion implies that, if the minimal type II error exponent is constrained to have $n D(\rho \| \sigma)$ as its first order term and $\sqrt{n} t_{2}$ as its second order term, then the minimal type I error is given by $\Phi\left(t_{2} / \sqrt{V(\rho \| \sigma)}\right)$. Hence the minimal asymptotic type I error varies smoothly between 0 and 1 as $t_{2}$ increases from $-\infty$ to $+\infty$ : see Figure 2, which is a plot of the function

$$
\alpha_{\infty}^{(2)}\left(t_{2}\right)=\inf \left\{\limsup _{n} \alpha\left(T_{n}\right) \mid-\liminf _{n} \frac{1}{\sqrt{n}}\left(\log \beta_{n}\left(T_{n}\right)+n D(\rho \| \sigma)\right) \geq t_{2}\right\} .
$$

The Gaussian c.d.f. $\Phi$ arises from the Central Limit Theorem (CLT), or rather from its 


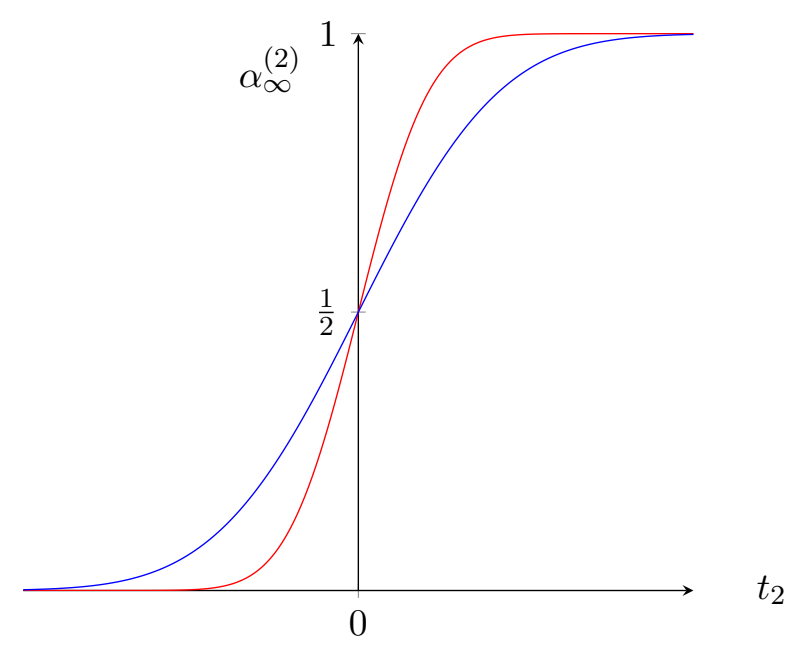

Figure 2: The minimal asymptotic type I error $\alpha_{\infty}^{(2)}\left(t_{2}\right)$, defined in Equation 1.4), for two different values of $V(\rho \| \sigma)$ (red: $V(\rho \| \sigma)=1$, blue: $V(\rho \| \sigma)=2$ ).

refinement, the Berry-Esseen Theorem (see e.g. [13]), which gives the rate of convergence of the distribution of the scaled sum of i.i.d. random variables to a normal distribution.

The study of second order asymptotics is a key step towards understanding a fundamental problem of both theoretical and practical interest, which is to determine how quickly the behaviour of a finite system approaches that of its asymptotic limit. Second order asymptotics have been obtained for various classical- ([42, 15, 46], ; see also the review [43] and references therein) and quantum information theoretic tasks (initiated in [44, 28]; see [27] and references therein for later works, as well as [47]). However, all results obtained in the quantum case pertain to the i.i.d. setting in which the underlying resource is assumed to be uncorrelated. In this paper, we obtain second order asymptotics for quantum settings beyond i.i.d. which had thus far remained a challenging, open problem ${ }^{2}$. Moreover, we do it for the task of quantum hypothesis testing which underlies various other information-theoretic tasks, such as the transmission of classical information through a quantum channel.

\section{Our contribution}

In this paper, we extend the study of second order asymptotics of the type II error exponent $\left(-\log \beta_{n}(\varepsilon)\right)$ of asymmmetric quantum hypothesis testing to settings beyond i.i.d.. In this case, the state $\omega_{n}$ which Bob receives is either $\rho_{n}$ or $\sigma_{n}$, but the latter are not necessarily of the tensor power (i.e. i.i.d.) form. More precisely, we consider two sequences $\hat{\rho}:=\left(\rho_{n}\right)_{n \in \mathbb{N}}$ and $\hat{\sigma}:=\left(\sigma_{n}\right)_{n \in \mathbb{N}}$, where for every $n, \rho_{n}$ and $\sigma_{n}$ are states (on a finite-dimensional Hilbert space $\mathcal{H}_{n}$ ) which need not be of the form $\rho^{\otimes n}$ and $\sigma^{\otimes n}$, respectively. We still assume, however, that for increasing $n$ the amount of information that can be extracted by performing a test increases linearly with a specified sequence of positive weights $\left(w_{n}\right)_{n \in \mathbb{N}}$, satisfying $w_{n} \rightarrow \infty$ as $n \rightarrow \infty$. In the case of $n$ i.i.d. copies, i.e. if $\rho_{n}=\rho^{\otimes n}$ and $\sigma_{n}=\sigma^{\otimes n}$, we choose

\footnotetext{
${ }^{2}$ It has been done for mixed source coding in 27] but there too the problem can essentially be reduced to the i.i.d. setting.
} 
$w_{n}=n$. Interesting examples of quantum states which fall within our setting are Gibbs states of quantum spin systems (with finite-range, translation-invariant interactions) at high temperature and quasi-free states of a fermionic lattice gas. The example of i.i.d. states is included in our setting and so we can recover the result of [44] and [28].

The first order asymptotics in this general setting was studied by Hiai, Mosonyi and Ogawa [20, where the authors introduced the analysis of the hypothesis testing problem using the existence and differentiability of the asymptotic logarithmic moment generating function, which leads to a proof of quantum Stein's lemma for various classes of correlated states (see [18], 24]). It is also worth mentioning that various extensions of quantum Stein's lemma for non i.i.d. states were previously treated in [7, 8, 19, 20, 22, 29, 30. Special cases of the second order asymptotics that we derive were obtained in [16] and [45]: in Lemma 26 of [45] the case in which $\hat{\rho}$ is a sequence of product states and $\hat{\sigma}$ is a sequence of i.i.d states was considered, whereas in [16] $\hat{\rho}$ was chosen to be i.i.d. while $\hat{\sigma}$ was taken to be some sequence of so-called universal states (see Lemma 1 of [16]).

Our main results are given by Theorem 1 and Corollary 1 of Section 3 . The essence of our strongest result (which is given by Corollary 1), can be easily conveyed through the example of quasi-free states of fermions on a one-dimensional lattice $\mathbb{Z}$. Suppose the sequence of states $\rho_{n}$ and $\sigma_{n}$ arising in our hypothesis testing problem are Gibbs states of these fermions, corresponding to the same Hamiltonian but at two different temperatures, restricted to a finite subset $\Lambda_{n}$ of the lattice. Even though these are non-i.i.d. states, we prove that the minimal type I error has the same functional dependence on the second order term of the type II error exponent as that for i.i.d. case shown in Figure 2. Note, however, that the relative entropy $D(\rho \| \sigma)$ is replaced by the relative entropy rate $d(\hat{\rho}, \hat{\sigma})$, the quantum information variance, $V(\rho \| \sigma)$, is replaced by the quantum information variance rate, $v(\hat{\rho}, \hat{\sigma})$ (defined in Equation (3.1) and Equation (3.2) respectively), and $n$ is replaced by the lattice size $\left|\Lambda_{n}\right|$.

\section{Notations and Definitions}

In this section let $\mathcal{H}$ denote a finite-dimensional Hilbert space, $\mathcal{B}(\mathcal{H})$ denote the algebra of linear operators acting on $\mathcal{H}$ and $\mathcal{B}_{s a}(\mathcal{H})$ denote the set of self-adjoint operators. Let $\mathcal{P}(\mathcal{H})$ be the set of positive semi-definite operators on $\mathcal{H}$ and $\mathcal{P}_{+}(\mathcal{H}) \subset \mathcal{P}(\mathcal{H})$ the set of (strictly) positive operators. Further, let $\mathcal{D}(\mathcal{H}):=\{\rho \in \mathcal{P}(\mathcal{H}) \mid \operatorname{Tr} \rho=1\}$ denote the set of density matrices on $\mathcal{H}$; we will use the terms "density matrix" and "state" interchangeably. We denote the support of an operator $A$ as $\operatorname{supp}(A)$ and the range of a projection operator $P$ as $\operatorname{ran}(P)$. Let $\mathbb{I} \in \mathcal{P}(\mathcal{H})$ denote the identity operator on $\mathcal{H}$, and id $: \mathcal{B}(\mathcal{H}) \mapsto \mathcal{B}(\mathcal{H})$ the identity map on operators on $\mathcal{H}$.

Any element $A$ of $\mathcal{B}_{\text {sa }}(\mathcal{H})$ has a spectral decomposition of the form $A=\sum_{\lambda \in \operatorname{sp}(A)} \lambda P_{\lambda}(A)$, where $\operatorname{sp}(A)$ denotes the spectrum of $A$, and $P_{\lambda}(A)$ is the projection operator corresponding to the eigenvalue $\lambda$. We denote by $A_{+}$the positive part of $A$. More precisely,

$$
A_{+}:=\sum_{\lambda \in \operatorname{sp}(A) \cap \mathbb{R}_{+}} \lambda P_{\lambda}(A)
$$

For any $\rho, \sigma \in \mathcal{P}(\mathcal{H})$, and for any $s \in[0,1]$, we define the following quantity which plays 
a central role in our proofs:

$$
\Psi_{s}(\rho \mid \sigma):=\log \operatorname{Tr}\left(\rho^{s} \sigma^{1-s}\right) .
$$

If $\rho$ and $\sigma$ have orthogonal supports, then $\Psi_{s}(\rho \mid \sigma)=-\infty$. Note that for $s \in[0,1)$,

$$
\Psi_{s}(\rho \mid \sigma)=(s-1) D_{s}(\rho \| \sigma),
$$

where $D_{s}(\rho \| \sigma)$ is the quantum relative Rényi entropy of order $s$. It is known that

$$
\lim _{s \rightarrow 1} D_{s}(\rho \| \sigma)=D(\rho \| \sigma)
$$

where $D(\rho \| \sigma)$ is the quantum relative entropy,

$$
D(\rho \| \sigma):=\operatorname{Tr} \rho(\log \rho-\log \sigma),
$$

if $\operatorname{supp}(\rho) \subseteq \operatorname{supp}(\sigma)$, and equal to $+\infty$ otherwise. Henceforth, we assume for simplicity that all states are faithful, so that $\operatorname{supp}(\rho)=\operatorname{supp}(\sigma)$. We also define the quantum information variance

$$
V(\rho \| \sigma):=\operatorname{Tr}\left(\rho(\log \rho-\log \sigma)^{2}\right)-D(\rho \| \sigma)^{2} .
$$

The following identities can be easily verified. :

Lemma 1. For any $\rho, \sigma \in \mathcal{D}(\mathcal{H})$ with $\operatorname{supp}(\rho)=\operatorname{supp}(\sigma)$ we have

$$
\left.\frac{\mathrm{d}}{\mathrm{d} s} \Psi_{s}(\rho \mid \sigma)\right|_{s=0}=-\left.D(\sigma \| \rho) \quad \frac{\mathrm{d}}{\mathrm{d} s} \Psi_{s}(\rho \mid \sigma)\right|_{s=1}=\left.D(\rho \| \sigma) \quad \frac{\mathrm{d}^{2}}{\mathrm{~d} s^{2}} \Psi_{s}(\rho \mid \sigma)\right|_{s=1}=V(\rho \| \sigma) .
$$

Moreover, for $s \in[0,1], \Psi_{s}^{\prime \prime}(\rho \mid \sigma) \geq 0$ and hence $\Psi_{s}(\rho \mid \sigma)$ is convex in $s$.

As in [24], we use relative modular operators in our proofs and intermediate results. Relative modular operators were introduced originally by Araki, and this allowed him to extend the notion of relative entropy to arbitrary states on a $C^{*}$-algebra (see [1, 2, 37]). We also refer to Petz's papers [38] and [39] for a discussion on the relation between the relative modular operator and Rényi divergences. We do not work with infinite-dimensional systems in the present paper; however, the language of modular theory will prove convenient even in the finite-dimensional case. In addition, extensions to infinite dimensions will be natural once statements and proofs are expressed in the language of modular theory (this will be done in a future paper). In particular the crucial relation $(2.10)$ (given below) will hold without change in the infinite-dimensional setting.

To define relative modular operators on a finite-dimensional operator algebra $\mathcal{B}(\mathcal{H})$, we start by equipping $\mathcal{A}=\mathcal{B}(\mathcal{H})$ with a Hilbert space structure through the Hilbert-Schmidt scalar product: for $A, B$ two elements of $\mathcal{A}$ we let $\langle A, B\rangle:=\operatorname{Tr} A^{*} B$. We define a map $\pi: \mathcal{B}(\mathcal{H}) \rightarrow \mathcal{B}(\mathcal{A})$ by $\pi(A): X \mapsto A X$, i.e. $\pi(A)$ is the map acting on $\mathcal{A}=\mathcal{B}(\mathcal{H})$ by left multiplication by $A$. This map is linear, one-to-one and has in addition the properties $\pi(A B)=\pi(A) \pi(B), \pi\left(A^{*}\right)=\pi(A)^{*}$, where here $\pi(A)^{*}$ denotes the adjoint of the map $\pi(A)$ defined through the relation $\langle X, \pi(A)(Y)\rangle=\left\langle\pi(A)^{*} X, Y\right\rangle$, and the following identity between operator norms $\|\pi(A)\|_{\mathcal{B}(\mathcal{A})}=\|A\|_{\mathcal{B}(\mathcal{H})}$. We will therefore identify $A$ with $\pi(A)$ and, because 
of the identity $\pi(A) X=A X$, will simply write $A$ for $\pi(A)$ (even though $\pi(A)$ is a linear map on $\mathcal{A}$, and $A$ is not!).

For any $\rho \in \mathcal{D}(\mathcal{H})$, we denote $\Omega_{\rho}:=\rho^{1 / 2} \in \mathcal{B}_{s a}(\mathcal{H})$. We then have the identity

$$
\operatorname{Tr}(\rho A)=\left\langle\Omega_{\rho}, A \Omega_{\rho}\right\rangle \quad \text { for all } A \in \mathcal{A}
$$

even though, again, the right-hand side should be written $\left\langle\Omega_{\rho}, \pi(A) \Omega_{\rho}\right\rangle$. This is nothing but a simple case of the well-known GNS representation (see e.g. Section 2.3.3 of [9]).

From now on, for simplicity of exposition, we only consider faithful states, i.e. any state $\rho$ will be such that $\operatorname{supp}(\rho)=\mathcal{H}$ and in particular any two states will satisfy $\operatorname{supp}(\rho)=\operatorname{supp}(\sigma)$. We then define the relative modular operator $\Delta_{\rho \mid \sigma}$ to be the map

$$
\begin{aligned}
\Delta_{\rho \mid \sigma}: \mathcal{A} & \rightarrow \mathcal{A} \\
A & \mapsto \rho A \sigma^{-1}
\end{aligned}
$$

Let us denote by $J$ the antilinear operator on $\mathcal{A}$ defined by $J: A \mapsto A^{*}$. This map is anti self-adjoint and anti-unitary:

$$
\forall A, B \in \mathcal{B}(\mathcal{H}), \quad\langle A, J(B)\rangle=\overline{\langle J(A), B\rangle}, \quad\langle J(A), J(B)\rangle=\overline{\langle A, B\rangle},
$$

and we easily obtain ${ }^{3}$ the relation $J \Delta_{\rho \mid \sigma} J=\Delta_{\sigma \mid \rho}^{-1}$. Note that 2.7) defines $\Delta_{\rho \mid \sigma}$ for any $\rho, \sigma$ in $\mathcal{P}_{+}(\mathcal{H})$ and not only for states.

As a linear operator on $\mathcal{B}(\mathcal{H}), \Delta_{\rho \mid \sigma}$ is positive and its spectrum $\operatorname{sp}\left(\Delta_{\rho \mid \sigma}\right)$ consists of the ratios of eigenvalues $\lambda / \mu, \lambda \in \operatorname{sp}(\rho), \mu \in \operatorname{sp}(\sigma)$. For any $x \in \operatorname{sp}\left(\Delta_{\rho \mid \sigma}\right)$, the corresponding spectral projection is the map

$$
\begin{aligned}
& P_{x}\left(\Delta_{\rho \mid \sigma}\right): \mathcal{A} \rightarrow \quad \mathcal{A} \\
& A \mapsto \sum_{\lambda \in \operatorname{sp}(\rho), \mu \in \operatorname{sp}(\sigma): \lambda / \mu=x} P_{\lambda}(\rho) A P_{\mu}(\sigma)
\end{aligned}
$$

Further, for any $s \in[0,1], \Delta_{\rho \mid \sigma}^{s}(A)=\rho^{s} A \sigma^{-s}$. For any $\rho, \sigma \in \mathcal{P}_{+}(\mathcal{H})$ and $s \in[0,1]$, the quantity $\Psi_{s}(\rho \mid \sigma)$ can be expressed in terms of the relative modular operator $\Delta_{\rho \mid \sigma}$ as follows

$$
\Psi_{s}(\rho \mid \sigma)=\log \left\langle\Omega_{\sigma}, \Delta_{\rho \mid \sigma}^{s} \Omega_{\sigma}\right\rangle, \quad \text { where } \Omega_{\sigma}:=\sigma^{1 / 2} .
$$

In addition, let $\mu_{\rho \mid \sigma}$ denote the spectral measure for $-\log \Delta_{\rho \mid \sigma}$ with respect to $\Omega_{\sigma}:=\sigma^{1 / 2}$, i.e. the probability measure such that for any bounded measurable function $f$,

$$
\left\langle\Omega_{\sigma}, f\left(-\log \Delta_{\rho \mid \sigma}\right) \Omega_{\sigma}\right\rangle=\int f(x) \mathrm{d} \mu_{\rho \mid \sigma}(x) \equiv \mathbb{E}[f(X)],
$$

where $X$ is a random variable of law $\mu_{\rho \mid \sigma}$ (see e.g. Sections VII and VIII of [40]). We then have in particular

$$
\Psi_{s}(\rho \mid \sigma)=\log \left(\int e^{-s x} \mathrm{~d} \mu_{\rho \mid \sigma}(x)\right) \equiv \log \mathbb{E}\left[e^{-s X}\right]
$$

\footnotetext{
${ }^{3}$ We can also derive the relation $J \Delta_{\rho \mid \sigma}^{1 / 2} A \Omega_{\sigma}=A^{*} \Omega_{\rho}$ for any $A \in \mathcal{A}$, which is precisely the relation defining $J$ and $\Delta_{\rho \mid \sigma}$ in the general algebraic case.
} 
The relation 2.12 plays a key role in our proof since it links the quantity $\Psi_{s}$ to the cumulant generating function of a classical random variable, and therefore allows us to employ the tools of classical probability theory. The quantity $\Psi_{s}$ can also be expressed in terms of the wellknown Nussbaum-Szkoła distributions (see [34]). For two density matrices $\rho, \sigma \in \mathcal{D}(\mathcal{H})$ with spectral decompositions

$$
\rho=\sum_{\lambda} \lambda P_{\lambda}(\rho), \quad \sigma=\sum_{\mu} \mu P_{\mu}(\sigma)
$$

these distributions are given by $\left(p_{\lambda, \mu}\right)_{\lambda, \mu}$ and $\left(q_{\lambda, \mu}\right)_{\lambda, \mu}$, where

$$
p_{\lambda, \mu}=\lambda \operatorname{Tr}\left(P_{\lambda}(\rho) P_{\mu}(\sigma)\right), \quad q_{\lambda, \mu}=\mu \operatorname{Tr}\left(P_{\lambda}(\rho) P_{\mu}(\sigma)\right) .
$$

There is of course a connection between the Nussbaum-Szkoła distributions and relative modular operators. Assume for simplicity that all ratios $\lambda / \mu$ are distinct and consider a random variable $Z$ which takes values $\lambda / \mu$ with probability $q_{\lambda, \mu}$. Then using 2.9) one can easily verify that

$$
\begin{aligned}
\mathbb{P}(Z=\lambda / \mu)=q_{\lambda, \mu} & =\mu \operatorname{Tr}\left(P_{\lambda}(\rho) P_{\mu}(\sigma)\right) \\
& =\left\langle\Omega_{\sigma}, P_{\lambda / \mu}\left(\Delta_{\rho \mid \sigma}\right) \Omega_{\sigma}\right\rangle \\
& =\left\langle\Omega_{\sigma}, \mathbf{1}_{\{\lambda / \mu\}}\left(\Delta_{\rho \mid \sigma}\right) \Omega_{\sigma}\right\rangle,
\end{aligned}
$$

where $\mathbf{1}_{\{\lambda / \mu\}}$ denotes the indicator function on the singleton $\{\lambda / \mu\}$, i.e. $\mathbf{1}_{\{\lambda / \mu\}}(x)$ is equal to 1 when $x=\lambda / \mu$ and equal to 0 else. This follows from the fact that, since $\Delta_{\rho \mid \sigma}$ is self-adjoint, the spectral theorem implies that $\mathbf{1}_{\{\lambda / \mu\}}\left(\Delta_{\rho \mid \sigma}\right)=\sum_{x \in \operatorname{sp}\left(\Delta_{\rho \mid \sigma}\right)} \mathbf{1}_{\{\lambda / \mu\}}(x) P_{x}\left(\Delta_{\rho \mid \sigma}\right)$. Equation (2.13) implies that for any bounded measurable function $f$,

$$
\mathbb{E}[f(Z)]=\left\langle\Omega_{\sigma}, f\left(\Delta_{\rho \mid \sigma}\right) \Omega_{\sigma}\right\rangle
$$

and hence the law of $Z$ is the law of $\Delta_{\rho \mid \sigma}$ with respect to $\Omega_{\sigma}:=\sigma^{1 / 2}$. This in turn implies that for any bounded measurable function $f$,

$$
\mathbb{E}[f(-\log Z)]=\left\langle\Omega_{\sigma}, f\left(-\log \Delta_{\rho \mid \sigma}\right) \Omega_{\sigma}\right\rangle .
$$

Hence the law of $-\log Z$ is precisely $\mu_{\rho \mid \sigma}$. As mentioned earlier, the advantage of the construction via modular operators is that it extends directly to the infinite-dimensional case.

\section{Second order asymptotics for hypothesis testing}

In this section, we state and prove our main results. Our general framework will be that of two sequences of states, $\hat{\rho}=\left(\rho_{n}\right)_{n \in \mathbb{N}}$ and $\hat{\sigma}=\left(\sigma_{n}\right)_{n \in \mathbb{N}}$, such that for every $n, \rho_{n}$ and $\sigma_{n}$ are elements of $\mathcal{D}\left(\mathcal{H}_{n}\right)$, where $\mathcal{H}_{n}$ is a finite-dimensional Hilbert space. We always assume that $\rho_{n}$ and $\sigma_{n}$ are faithful, that is, $\operatorname{supp}\left(\rho_{n}\right)=\operatorname{supp}\left(\sigma_{n}\right)=\mathcal{H}_{n}$. We also fix a sequence of weights $\hat{w}=\left(w_{n}\right)_{n \in \mathbb{N}}$ which we assume is an increasing sequence of positive numbers satisfying $\lim _{n \rightarrow \infty} w_{n}=\infty$.

The quantity whose second order asymptotic expansion we wish to evaluate is the type II error exponent, which is given for any $\varepsilon \in(0,1)$ by $-\log \beta_{n}(\varepsilon)$, where

$$
\beta_{n}(\varepsilon):=\inf _{0 \leq T_{n} \leq \mathbb{I}_{n}}\left\{\beta\left(T_{n}\right) \mid \alpha\left(T_{n}\right) \leq \varepsilon\right\} .
$$


Here $\alpha\left(T_{n}\right):=\operatorname{Tr}\left(\left(\mathbb{I}_{n}-T_{n}\right) \rho_{n}\right)$ and $\beta\left(T_{n}\right):=\operatorname{Tr}\left(T_{n} \sigma_{n}\right)$, are the type $I$ and type $I I$ errors, in testing $\rho_{n}$ vs. $\sigma_{n}$ and $\mathbb{I}_{n}$ is the identity operator acting on $\mathcal{H}_{n}$.

Remark 1. We restrict our results to the case of faithful states $\rho_{n}, \sigma_{n}$ only to make our exposition more transparent. Simple limiting arguments show that all our results remain valid in the case in which $\operatorname{supp}\left(\rho_{n}\right) \subseteq \operatorname{supp}\left(\sigma_{n}\right)$.

Our main results hold under the following conditions on the sequences of states $\left(\rho_{n}, \sigma_{n}\right)_{n \in \mathbb{N}}$ with respect to the weights $\left(w_{n}\right)_{n \in \mathbb{N}}$ :

Condition 1. There exists $r>0$ such that

1. for every $n$, the function $E_{n}(z)=\Psi_{1-z}\left(\rho_{n} \mid \sigma_{n}\right)$ originally defined for $z \in[0,1]$ can be extended to an analytic function in the complex open ball $B_{\mathbb{C}}(0, r)$,

2. for every $x$ in the real open ball $B_{\mathbb{R}}(0, r)$, the following limit exists:

$$
E(x)=\lim _{n \rightarrow \infty} \frac{1}{w_{n}} E_{n}(x),
$$

3. one has the uniform bound

$$
\sup _{n \in \mathbb{N}} \sup _{z \in B_{\mathbb{C}}(0, r)} \frac{1}{w_{n}}\left|E_{n}(z)\right|<+\infty .
$$

Vitali's Theorem, stated in Appendix A, implies that, if 1 holds, then $E$ can be extended to an analytic function on $B_{\mathbb{C}}(0, r)$, and the derivatives $\frac{1}{w_{n}} E_{n}^{\prime}(0)$ and $\frac{1}{w_{n}} E_{n}^{\prime \prime}(0)$ converge to $E^{\prime}(0)$ and $E^{\prime \prime}(0)$ respectively. By Lemma 1, this shows that the following definition is meaningful under 1:

Definition 1. Let $\hat{\rho}=\left(\rho_{n}\right)_{n \in \mathbb{N}}$ and $\hat{\sigma}=\left(\sigma_{n}\right)_{n \in \mathbb{N}}$ denote two sequences of states that satisfy 1 with respect to a sequence of weights $\left(w_{n}\right)_{n \in \mathbb{N}}$. Their quantum relative entropy rate is defined as

$$
d(\hat{\rho}, \hat{\sigma}):=\lim _{n \rightarrow \infty} \frac{1}{w_{n}} D\left(\rho_{n} \| \sigma_{n}\right)
$$

and their quantum information variance rate is defined as

$$
v(\hat{\rho}, \hat{\sigma}):=\lim _{n \rightarrow \infty} \frac{1}{w_{n}} V\left(\rho_{n} \| \sigma_{n}\right) .
$$

Our main result on second order asymptotics is given by the following theorem, which we prove in Section 3.2 .

Theorem 1. Fix $\varepsilon \in(0,1)$, and let $\hat{\rho}=\left(\rho_{n}\right)_{n \in \mathbb{N}}$ and $\hat{\sigma}=\left(\sigma_{n}\right)_{n \in \mathbb{N}}$ denote two sequences of states that satisfy 1 with respect to a sequence of weights $\left(w_{n}\right)_{n \in \mathbb{N}}$. Define

$$
t_{2}^{*}(\varepsilon)=\sqrt{v(\hat{\rho}, \hat{\sigma})} \Phi^{-1}(\varepsilon)
$$

where $\Phi$ is the cumulative distribution function of a standard normal distribution and $v(\hat{\rho}, \hat{\sigma})$ is the quantum information variance rate defined through Equation (3.2). Then for any $t_{2}>t_{2}^{*}(\varepsilon)$ there exists a function $f_{t_{2}}(x) \underset{+\infty}{=} o(\sqrt{x})$ such that for any $n \in \mathbb{N}$ :

$$
-\log \beta_{n}(\varepsilon) \leq D\left(\rho_{n} \| \sigma_{n}\right)+\sqrt{w_{n}} t_{2}+f_{t_{2}}\left(w_{n}\right),
$$


and for any $t_{2}<t_{2}^{*}(\varepsilon)$, any function $f(x) \underset{+\infty}{=} o(\sqrt{x})$, for $n$ large enough:

$$
-\log \beta_{n}(\varepsilon) \geq D\left(\rho_{n} \| \sigma_{n}\right)+\sqrt{w_{n}} t_{2}+f\left(w_{n}\right) .
$$

Let us comment on the above theorem, and explain why it captures the second order behaviour of $-\log \beta_{n}(\varepsilon)$. For this discussion, let us denote

$$
\triangle_{n} D=D\left(\rho_{n} \| \sigma_{n}\right)-w_{n} d(\hat{\rho}, \hat{\sigma})
$$

By Definition 1 we know that $D\left(\rho_{n} \| \sigma_{n}\right)=w_{n} d(\hat{\rho}, \hat{\sigma})+o\left(w_{n}\right)$, so that $-\log \beta_{n}(\varepsilon)=w_{n} d(\hat{\rho}, \hat{\sigma})+$ $o\left(w_{n}\right)$. The second order term of $-\log \beta_{n}(\varepsilon)$ will therefore depend on the relative magnitude of $\triangle_{n} D$ and $\sqrt{w_{n}}$. The simplest situation is summarized in the following corollary.

Corollary 1. Fix $\varepsilon \in(0,1)$, and let $\hat{\rho}=\left(\rho_{n}\right)_{n \in \mathbb{N}}$ and $\hat{\sigma}=\left(\sigma_{n}\right)_{n \in \mathbb{N}}$ denote two sequences of states that satisfy 1 with respect to a sequence of weights $\left(w_{n}\right)_{n \in \mathbb{N}}$. In addition, assume that

$$
D\left(\rho_{n} \| \sigma_{n}\right)=w_{n} d(\hat{\rho}, \hat{\sigma})+o\left(\sqrt{w_{n}}\right) .
$$

Let

$$
t_{2}^{*}(\varepsilon):=\sqrt{v(\hat{\rho}, \hat{\sigma})} \Phi^{-1}(\varepsilon) .
$$

Then for any $t_{2}>t_{2}^{*}(\varepsilon)$ there exists a function $f_{t_{2}}(x) \underset{+\infty}{=} o(\sqrt{x})$ such that for any $n \in \mathbb{N}$ :

$$
-\log \beta_{n}(\varepsilon) \leq w_{n} d(\hat{\rho}, \hat{\sigma})+\sqrt{w_{n}} t_{2}+f_{t_{2}}\left(w_{n}\right),
$$

and for any $t_{2}<t_{2}^{*}(\varepsilon)$, any function $f(x) \underset{+\infty}{=} o(\sqrt{x})$, for $n$ large enough:

$$
-\log \beta_{n}(\varepsilon) \geq w_{n} d(\hat{\rho}, \hat{\sigma})+\sqrt{w_{n}} t_{2}+f\left(w_{n}\right) .
$$

The proof of Corollary 1 is immediate from Theorem 1 . It says that if $\left(\rho_{n}, \sigma_{n}\right)_{n \in \mathbb{N}}$ satisfies 1 with respect to a sequence of weights $\left(w_{n}\right)_{n \in \mathbb{N}}$ and $\triangle_{n} D=o\left(\sqrt{w_{n}}\right)$, then for any $\delta>0$, we have

$w_{n} d(\hat{\rho}, \hat{\sigma})+\sqrt{w_{n}}\left(t_{2}^{*}(\varepsilon)-\delta\right)+o\left(\sqrt{w_{n}}\right) \leq-\log \beta_{n}(\varepsilon) \leq w_{n} d(\hat{\rho}, \hat{\sigma})+\sqrt{w_{n}}\left(t_{2}^{*}(\varepsilon)+\delta\right)+o\left(\sqrt{w_{n}}\right)$.

If on the other extreme $\sqrt{w_{n}}=o\left(\triangle_{n} D\right)$ then it is

$$
-\log \beta_{n}(\varepsilon)=w_{n} d(\hat{\rho}, \hat{\sigma})+\triangle_{n} D+o\left(\triangle_{n} D\right)
$$

that captures the second order of $-\log \beta_{n}(\varepsilon)$.

Remark 2. An equivalent statement to the above results is the following: define for $t_{2} \in \mathbb{R}$

$$
\begin{aligned}
& \tilde{\alpha}_{\infty}^{(2)}\left(t_{2}\right)=\inf \left\{\limsup _{n} \alpha\left(T_{n}\right) \mid-\liminf _{n} \frac{1}{\sqrt{w_{n}}}\left(\log \beta\left(T_{n}\right)+D\left(\rho_{n} \| \sigma_{n}\right)\right) \geq t_{2} ; 0 \leq T_{n} \leq \mathbb{I}_{n}\right\}, \\
& \alpha_{\infty}^{(2)}\left(t_{2}\right)=\inf \left\{\limsup _{n} \alpha\left(T_{n}\right) \mid-\liminf _{n} \frac{1}{\sqrt{w_{n}}}\left(\log \beta\left(T_{n}\right)+w_{n} d(\hat{\rho}, \hat{\sigma})\right) \geq t_{2} ; 0 \leq T_{n} \leq \mathbb{I}_{n}\right\} .
\end{aligned}
$$

Then, under the assumptions of Theorem 1 (respectively Corollary 1), one has

$$
\tilde{\alpha}_{\infty}^{(2)}\left(t_{2}\right)=\Phi\left(t_{2} / \sqrt{v(\hat{\rho}, \hat{\sigma})}\right) \quad\left(\text { respectively } \alpha_{\infty}^{(2)}\left(t_{2}\right)=\Phi\left(t_{2} / \sqrt{v(\hat{\rho}, \hat{\sigma})}\right)\right) .
$$




\subsection{Ingredients of the proof of Theorem 1}

A theorem proved by Bryc [1] (stated as Theorem 2 below), the lower bound in Lemma 2 and Proposition 1 are the ingredients required to prove Theorem 1. Bryc's theorem can be viewed as a generalization of the Central Limit Theorem for sequences of random variables which are not necessarily i.i.d. It was proven originally in [11. Lemma 2 involves bounds on the minimum error probability of symmetric hypothesis testing and was proven in [24].

The following version of Bryc's theorem is adapted from [25]. A full proof can be found in Appendix A.

Theorem 2 (Bryc's theorem). Let $\left(X_{n}\right)_{n \in \mathbb{N}}$ be a sequence of random variables, and $\left(w_{n}\right)_{n \in \mathbb{N}}$ a sequence of weights, i.e. positive numbers satisfying $\lim _{n \rightarrow \infty} w_{n}=\infty$. If there exists $r>0$ such that the following conditions hold:

- for any $n \in \mathbb{N}$, the function $H_{n}(z):=\log \mathbb{E}\left[\mathrm{e}^{-z X_{n}}\right]$ is analytic in the complex open ball $B_{\mathbb{C}}(0, r)$,

- the limit $H(x)=\lim _{n \rightarrow \infty} \frac{1}{w_{n}} H_{n}(x)$ exists for any $x \in(-r, r)$,

- we have the uniform bound $\sup _{n \in \mathbb{N}} \sup _{z \in B_{\mathbb{C}}(0, r)} \frac{1}{w_{n}}\left|H_{n}(z)\right|<+\infty$,

then $H$ is analytic on $B_{\mathbb{C}}(0, r)$ and we have the convergence in distribution ${ }^{4}$ as $n \rightarrow \infty$

$$
\frac{X_{n}+H_{n}^{\prime}(0)}{\sqrt{w_{n}}} \stackrel{\mathrm{d}}{\longrightarrow} \mathcal{N}\left(0, H^{\prime \prime}(0)\right),
$$

Remark 3. If $X_{n}$ is of the form $X_{n}=A_{1}+\ldots+A_{n}$ for a sequence $\left(A_{n}\right)_{n \in \mathbb{N}}$ of independent, identically distributed random variables, then for any $n$ we have $\frac{1}{n} H_{n}(z)=H(z)$. Therefore, the conditions of the above theorem are satisfied (with respect to the weights $w_{n}=n$ ) as soon as $H(z)$ can be extended to an analytic function in a complex neighbourhood of the origin. Note that this is a stronger condition than the existence of a second moment.

Let us comment on the use we make of Theorem 2. Let $\hat{\rho}=\left(\rho_{n}\right)_{n \in \mathbb{N}}$ and $\hat{\sigma}=\left(\sigma_{n}\right)_{n \in \mathbb{N}}$ denote two sequences of states satisfying 1 . Setting $s=1-z$, with $z \in[0,1]$ in Equation (2.12) and using the cyclicity of the trace function we obtain for $\Omega_{\rho_{n}}:=\rho_{n}^{1 / 2}$

$$
\Psi_{1-z}\left(\rho_{n} \mid \sigma_{n}\right)=\log \left\langle\Omega_{\rho_{n}}, \Delta_{\sigma_{n} \mid \rho_{n}}^{z} \Omega_{\rho_{n}}\right\rangle=\log \mathbb{E}\left[\mathrm{e}^{-z X_{n}}\right]
$$

where $\left(X_{n}\right)_{n \in \mathbb{N}}$ denotes a sequence of random variables with law $\mu_{\sigma_{n}} \mid \rho_{n}$, defined analogously to Equation (2.11). Then we have

$$
H_{n}(z):=\log \mathbb{E}\left[e^{-z X_{n}}\right]=\Psi_{1-z}\left(\rho_{n} \mid \sigma_{n}\right)
$$

and 1 implies that $\left(X_{n}\right)_{n \in \mathbb{N}}$ satisfies the assumptions of Bryc's theorem. Since

$$
\begin{gathered}
H_{n}^{\prime}(0)=-\left.\frac{\mathrm{d}}{\mathrm{d} z} \Psi_{z}\left(\rho_{n} \mid \sigma_{n}\right)\right|_{z=1}=-D\left(\rho_{n} \| \sigma_{n}\right), \\
H_{n}^{\prime \prime}(0)=\left.\frac{\mathrm{d}^{2}}{\mathrm{~d} z^{2}} \Psi_{z}\left(\rho_{n} \mid \sigma_{n}\right)\right|_{z=1}=V\left(\rho_{n} \| \sigma_{n}\right),
\end{gathered}
$$

\footnotetext{
${ }^{4}$ We use the notation $\mathcal{N}\left(\mu, \sigma^{2}\right)$ to denote a normal distribution of mean $\mu$ and variance $\sigma^{2}$.
} 
1 and Theorem 2 imply the convergence in distribution

$$
Y_{n}:=\frac{X_{n}-D\left(\rho_{n} \| \sigma_{n}\right)}{\sqrt{w_{n}}} \stackrel{\mathrm{d}}{\longrightarrow} \mathcal{N}(0, v(\hat{\rho}, \hat{\sigma})) .
$$

The above convergence (3.8) plays a fundamental role in our proof.

Another key ingredient of our proof is Lemma 2, which gives an upper bound and a lower bound on the minimum total probability of error in symmetric hypothesis testing. If the states corresponding to the two hypotheses are $\rho$ and $\sigma$, then the latter is defined as:

$$
e_{\mathrm{sym}}^{*}(\rho, \sigma):=\inf _{0 \leq T \leq \mathbb{I}} e_{\mathrm{sym}}(\rho, \sigma, T),
$$

where

$$
e_{\mathrm{sym}}(\rho, \sigma, T):=\alpha(T)+\beta(T),
$$

denotes the total probability of error under a test $T$.

Remark 4. In the Bayesian setting, one can assign prior probabilities to the two states, i.e. assume that the state is $\rho$ with probability $p$, and $\sigma$ with probability $(1-p)$ for some $p \in(0,1)$. The aim is then to minimize the quantity $[p \operatorname{Tr}((\mathbb{I}-T) \rho)+(1-p) \operatorname{Tr}(T \sigma)]$, which is $e_{\text {sym }}(p \rho,(1-p) \sigma, T)$. For convenience we absorb the scalar factors $p$ and $(1-p)$ into $\rho$ and $\sigma$, so that the latter are no longer of trace 1 . In order to accommodate this scenario, as well as for particular applications in the proof of our main result, in the following we consider the quantities $e_{\mathrm{sym}}^{*}(A, B)$ and $e_{\mathrm{sym}}(A, B)$ for arbitrary operators $A, B \in \mathcal{P}_{+}(\mathcal{H})$. We also use the fact that $e_{\mathrm{sym}}^{*}$ is symmetric in its arguments, i.e. $e_{\mathrm{sym}}^{*}(A, B)=e_{\mathrm{sym}}^{*}(B, A)$.

The infimum in Equation $(3.9)$ is attained for a test given by the projector $(A-B)_{+}$, as is given by the quantum extension of the Neyman-Pearson lemma [23, 17] which states that for any $A, B \in \mathcal{P}_{+}(\mathcal{H})$

$$
\mathrm{e}_{\text {sym }}^{*}(A, B)=\mathrm{e}_{\mathrm{sym}}\left(A, B,(A-B)_{+}\right) \text {. }
$$

For any $\gamma \in \mathbb{R}$, the orthogonal projection $T^{\gamma}:=\left(A-e^{\gamma} B\right)_{+}$onto the positive part of $\left(A-e^{\gamma} B\right)$, is called the quantum Neyman-Pearson test for $A$ vs. $B$, of rate $\gamma$. Using Equation (3.10), one can show that the tests $T^{\gamma}$ are optimal in the sense that for any other test $T^{\prime}$,

$$
\alpha\left(T^{\prime}\right) \leq \alpha\left(T^{\gamma}\right) \Rightarrow \beta\left(T^{\prime}\right) \geq \beta\left(T^{\gamma}\right)
$$

In the classical framework, i.e. when $[\rho, \sigma]=0$ then for any $\gamma \in \mathbb{R}, T^{\gamma}$ is $P_{\mathbb{R}_{+}}\left(\log \left(\rho \sigma^{-1}\right)-\gamma \mathbb{I}\right)$, and we recover the Neyman-Pearson tests considered in classical hypothesis testing. More precisely, writing $\rho$ and $\sigma$ in the basis in which they are both diagonal, so that their spectral decompositions read

$$
\rho=\sum_{i} \lambda_{i} P_{i} \quad \mu=\sum_{i} \mu_{i} P_{i},
$$

we have

$$
T^{\gamma}=\sum_{i \mid \log \left(\lambda_{i} / \mu_{i}\right) \geq \gamma} P_{i}
$$


and

$$
\beta\left(T^{\gamma}\right)=\sum_{i \mid \log \left(\lambda_{i} / \mu_{i}\right) \geq \gamma} \mu_{i}=\mathbb{P}(X \geq \gamma)
$$

where $X$ is a random variable which takes values $\log \left(\lambda_{i} / \mu_{i}\right)$ with probabilities $\mu_{i}$. This is particularly useful in the i.i.d. setting in which we have $n$ identical copies of $\rho$ and $\sigma$. In this case the random variable $X \equiv X_{n}$ can be expressed as a sum of $n$ i.i.d. random variables, and we can use the full power of probability theory 5 . However, this equivalence between quantum and classical Neyman-Pearson tests is not valid in the non-commutative case, since $\left(\rho-e^{\gamma} \sigma\right)_{+} \neq\left(\log \left(\rho \sigma^{-1}\right)-\gamma \mathbb{I}\right)_{+}$.

The following lemma from [24] (whose proof we include in Appendix B), allows us to circumvent this problem; even when $[\rho, \sigma] \neq 0$, it provides bounds on $e_{\mathrm{sym}}^{*}(\rho, \sigma)$ in terms of expectations of a classical random variable, which, in the case of $n$ identical copies of the underlying state, can be expressed as a sum of $n$ i.i.d. random variables as above. We only use the lower bound on $e_{\mathrm{sym}}^{*}(\rho, \sigma)$ in proving Theorem 1 , but also state the upper bound for sake of completeness.

Lemma 2. For any $A, B \in \mathcal{P}_{+}(\mathcal{H})$ and $s \in[0,1]$ :

$$
\left\langle\Omega_{B}, \Delta_{A \mid B}\left(1+\Delta_{A \mid B}\right)^{-1} \Omega_{B}\right\rangle \leq \mathrm{e}_{\mathrm{sym}}^{*}(A, B) \leq\left\langle\Omega_{B}, \Delta_{A \mid B}^{s} \Omega_{B}\right\rangle .
$$

where

$$
\begin{aligned}
\left\langle\Omega_{B}, \Delta_{A \mid B}\left(1+\Delta_{A \mid B}\right)^{-1} \Omega_{B}\right\rangle & =\sum_{(\lambda, \mu) \in \operatorname{sp}(A) \times \operatorname{sp}(B)} \frac{\lambda / \mu}{1+\lambda / \mu} \operatorname{Tr}\left(B P_{\lambda}(A) P_{\mu}(B)\right), \\
\left\langle\Omega_{B}, \Delta_{A \mid B}^{s} \Omega_{B}\right\rangle & =\operatorname{Tr} A^{s} B^{1-s} .
\end{aligned}
$$

Equivalently,

$$
\mathbb{E}\left[\frac{1}{1+e^{X}}\right] \leq \mathrm{e}_{\mathrm{sym}}^{*}(A, B) \leq \mathbb{E}\left[e^{-s X}\right],
$$

where $X$ is a random variable with law $\mu_{A \mid B}$ (defined through (2.11)).

Proof. See Appendix B.

The following result follows immediately from Lemma 2 , and is also proven in Appendix B.

Corollary 2. For any $\rho, \sigma \in \mathcal{P}_{+}(\mathcal{H})$ and any $v, \theta \in \mathbb{R}$,

$$
\mathrm{e}_{\mathrm{sym}}^{*}\left(\sigma, \rho \mathrm{e}^{-\theta}\right) \geq \frac{\mathrm{e}^{-\theta}}{1+\mathrm{e}^{v-\theta}} \mathbb{P}(X \leq v)
$$

where $X$ is a random variable with law $\mu_{\sigma \mid \rho}$ defined similarly to Equation (2.11).

The last ingredient in the proof of Theorem 1 is the following explicit construction, which is due to $\mathrm{Li}$ (in [28]). We give a full proof in Appendix C.

\footnotetext{
${ }^{5}\left(-X_{n}\right)$ is known as the observed log-likelihood ratio or information content random variable and can be used to define the classical Neyman-Pearson tests.
} 
Proposition 1. Let $\rho, \sigma$ be two states in $\mathcal{D}(\mathcal{H})$. For any $L>0$ there exists a test $T$ such that

$$
\operatorname{Tr} \rho(1-T) \leq\left\langle\Omega_{\rho}, P_{[0, L)}\left(\Delta_{\rho \mid \sigma}\right) \Omega_{\rho}\right\rangle \quad \text { and } \quad \operatorname{Tr} \sigma T \leq L^{-1},
$$

where $P_{[0, L)}\left(\Delta_{\rho \mid \sigma}\right):=\sum_{x \in[0, L)} P_{x}\left(\Delta_{\rho \mid \sigma}\right)$, with $P_{x}\left(\Delta_{\rho \mid \sigma}\right)$ being the projection operator defined through (2.9).

\subsection{Proof of Theorem 1}

The proof is divided into two parts: Equation (3.3) and Equation (3.4).

Proof of Equation (3.3) By continuity and monotonicity of $\Phi$, it suffices to prove that for any $\delta>0$, there exists $g_{\delta}(x) \underset{+\infty}{=} o(\sqrt{x})$ such that for any $n \in \mathbb{N}$ :

$$
-\log \beta_{n}(\varepsilon) \leq D\left(\rho_{n} \| \sigma_{n}\right)+\sqrt{w_{n} v(\hat{\rho} \| \hat{\sigma})} \Phi^{-1}(\varepsilon+\delta)+o\left(\sqrt{w_{n}}\right) .
$$

For any test $T_{n}$, and real numbers $\theta_{n}$ and $v_{n}$ (to be specified later), Corollary 2 as well as the symmetry of $e_{\text {sym }}^{*}$ give

$$
\mathrm{e}^{-\theta_{n}} \operatorname{Tr}\left(\left(\mathbb{I}_{n}-T_{n}\right) \rho_{n}\right)+\operatorname{Tr}\left(T_{n} \sigma_{n}\right) \geq e_{s y m}^{*}\left(\sigma_{n}, \rho_{n} \mathrm{e}^{-\theta_{n}}\right) \geq \frac{\mathrm{e}^{-\theta_{n}}}{1+\mathrm{e}^{v_{n}-\theta_{n}}} \mathbb{P}\left(X_{n} \leq v_{n}\right),
$$

where $X_{n}$ is a random variable of law $\mu_{\sigma_{n} \mid \rho_{n}}$. Hence, for any sequence of tests $\left(T_{n}\right)_{n \in \mathbb{N}}$ such that $\operatorname{Tr}\left(\left(\mathbb{I}_{n}-T_{n}\right) \rho_{n}\right) \leq \varepsilon$, we have

$$
\begin{aligned}
\operatorname{Tr}\left(T_{n} \sigma_{n}\right) & \geq \mathrm{e}^{-\theta_{n}}\left(\frac{\mathbb{P}\left(X_{n} \leq v_{n}\right)}{1+\mathrm{e}^{v_{n}-\theta_{n}}}-\operatorname{Tr}\left(\left(\mathbb{I}_{n}-T_{n}\right) \rho_{n}\right)\right) \\
& \geq \mathrm{e}^{-\theta_{n}}\left(\frac{\mathbb{P}\left(X_{n} \leq v_{n}\right)}{1+\mathrm{e}^{v_{n}-\theta_{n}}}-\varepsilon\right) .
\end{aligned}
$$

Then by relation (3.8) and Equation (3.2), we know that the random variable

$$
Y_{n}:=\frac{X_{n}-D\left(\rho_{n} \| \sigma_{n}\right)}{\sqrt{w_{n} v(\hat{\rho}, \hat{\sigma})}}
$$

converges in law to a standard normal distribution. Let us choose

$$
v_{n}:=D\left(\rho_{n} \| \sigma_{n}\right)+\sqrt{w_{n} v(\hat{\rho}, \hat{\sigma})} \Phi^{-1}(\varepsilon+\delta),
$$

with $\delta>0$, so that:

$$
\mathbb{P}\left(X_{n} \leq v_{n}\right)=\mathbb{P}\left(Y_{n} \leq \Phi^{-1}(\varepsilon+\delta)\right) \underset{n \rightarrow \infty}{\rightarrow} \Phi\left(\Phi^{-1}(\varepsilon+\delta)\right)=\varepsilon+\delta>\varepsilon .
$$

Therefore, there exists $\varepsilon^{\prime}>\varepsilon$ and $n_{0} \in \mathbb{N}$ such that for all $n \geq n_{0}, \mathbb{P}\left(X_{n} \leq v_{n}\right) \geq \varepsilon^{\prime}>\varepsilon$. Take $\theta_{n}:=v_{n}+h\left(w_{n}\right)$, for some positive function $h$ such that $h(x) \underset{+\infty}{=} o(\sqrt{x})$ but $h\left(w_{n}\right) \rightarrow \infty$ as $n \rightarrow \infty$. Hence, again for $n$ large enough,

$$
\mathrm{e}^{-\theta_{n}}\left(\frac{\mathbb{P}\left(X_{n} \leq v_{n}\right)}{1+\mathrm{e}^{v_{n}-\theta_{n}}}-\varepsilon\right) \geq \mathrm{e}^{-\theta_{n}}\left(\varepsilon^{\prime}\left(1+e^{-h\left(w_{n}\right)}\right)^{-1}-\varepsilon\right)
$$


and since the quantity in parenthesis on the right hand side of the above inequality is bounded below, and (3.16) is true for any sequence of tests $\left(T_{n}\right)_{n \in \mathbb{N}}$ such that $\operatorname{Tr}\left(\rho_{n}\left(\mathbb{I}_{n}-T_{n}\right)\right) \leq \varepsilon$, we have for $n$ large enough

$$
-\log \beta_{n}(\varepsilon) \leq D\left(\rho_{n} \| \sigma_{n}\right)+\sqrt{w_{n} v(\hat{\rho}, \hat{\sigma})} \Phi^{-1}(\varepsilon+\delta)+\tilde{g}_{\delta}\left(w_{n}\right),
$$

with $\tilde{g}_{\delta} \underset{+\infty}{=} o(\sqrt{x})$, and modifying $\tilde{g}_{\delta}$ for a finite number of values gives the desired inequality.

Proof of Equation (3.4) For each $n$ in $\mathbb{N}$, let

$$
L_{n}=\exp \left(D\left(\rho_{n} \| \sigma_{n}\right)+\sqrt{w_{n}} t_{2}+f\left(w_{n}\right)\right)
$$

with $f(x) \underset{+\infty}{=} o(\sqrt{x})$, and consider the test $T_{n}$ associated by Proposition 1 with $L_{n}$. Then relations 3.14 imply

$$
-\log \beta\left(T_{n}\right) \geq D\left(\rho_{n} \| \sigma_{n}\right)+\sqrt{w_{n}} t_{2}+f\left(w_{n}\right)
$$

and

$$
\begin{aligned}
\alpha\left(T_{n}\right) & \leq\left\langle\Omega_{\rho_{n}}, P_{\left(0, L_{n}\right)}\left(\Delta_{\rho_{n} \mid \sigma_{n}}\right) \Omega_{\rho_{n}}\right\rangle, \\
& \leq\left\langle\Omega_{\rho_{n}}, P_{\left(-\infty, \log L_{n}\right)}\left(\log \Delta_{\rho_{n} \mid \sigma_{n}}\right) \Omega_{\rho_{n}}\right\rangle .
\end{aligned}
$$

As we remarked after definition (2.7), $J \Delta_{\rho \mid \sigma} J=\Delta_{\sigma \mid \rho}^{-1}$, with $J$ being the map : $A \mapsto A^{*}$. This, together with the fact that $J^{2}=$ id implies that $\log \Delta_{\rho_{n} \mid \sigma_{n}}=-J\left(\log \Delta_{\sigma_{n} \mid \rho_{n}}\right) J$, and we obtain

$$
P_{\left(-\infty, \log L_{n}\right)}\left(\log \Delta_{\rho_{n} \mid \sigma_{n}}\right)=P_{\left(-\infty, \log L_{n}\right)}\left(-J \log \Delta_{\sigma_{n} \mid \rho_{n}} J\right)=J P_{\left(-\infty, \log L_{n}\right)}\left(-\log \Delta_{\sigma_{n} \mid \rho_{n}}\right) J .
$$

Noting that $J$ is anti self-adjoint $(2.8), J \Omega_{\rho_{n}}=\Omega_{\rho_{n}}$, and the definition of the random variable $X_{n} \sim \mu_{\sigma_{n} \mid \rho_{n}}$, we obtain

$$
\begin{aligned}
\alpha\left(T_{n}\right) & \leq\left\langle\Omega_{\rho_{n}}, P_{\left(-\infty, \log L_{n}\right)}\left(\log \Delta_{\rho_{n} \mid \sigma_{n}}\right) \Omega_{\rho_{n}}\right\rangle \\
& =\left\langle\Omega_{\rho_{n}}, J P_{\left(-\infty, \log L_{n}\right)}\left(-\log \Delta_{\sigma_{n} \mid \rho_{n}}\right) J \Omega_{\rho_{n}}\right\rangle \\
& =\left\langle J \Omega_{\rho_{n}}, P_{\left(-\infty, \log L_{n}\right)}\left(-\log \Delta_{\sigma_{n}} \mid \rho_{n}\right) J \Omega_{\rho_{n}}\right\rangle \\
& =\left\langle\Omega_{\rho_{n}}, P_{\left(-\infty, \log L_{n}\right)}\left(-\log \Delta_{\sigma_{n} \mid \rho_{n}}\right) \Omega_{\rho_{n}}\right\rangle \\
& =\mathbb{P}\left(X_{n} \leq \log L_{n}\right)=\mathbb{P}\left(\frac{X_{n}-D\left(\rho_{n}|| \sigma_{n}\right)}{\sqrt{w_{n}}} \leq t_{2}+\frac{f\left(w_{n}\right)}{\sqrt{w_{n}}}\right) .
\end{aligned}
$$

By consequence (3.8) of Bryc's theorem, the above converges to $\Phi\left(t_{2} / \sqrt{v(\hat{\rho}, \hat{\sigma})}\right)<\varepsilon$. Therefore, for $n$ large enough, one has $\alpha\left(T_{n}\right) \leq \varepsilon$. The bound (3.18) proves Equation (3.4).

\section{Examples}

In this section we give examples of sequences of quantum states, $\hat{\rho}=\left(\rho_{n}\right)_{n \in \mathbb{N}}$ and $\hat{\sigma}=$ $\left(\sigma_{n}\right)_{n \in \mathbb{N}}$, for which 1 holds, and hence Theorem 1 applies. Our examples closely follow those from [24], since 1 is stronger than the conditions required for the (first order) Stein lemma given in that reference. 


\subsection{Quantum i.i.d. states}

The simplest example is that of i.i.d. states: $\rho_{n}=\rho^{\otimes n}$ and $\sigma_{n}=\sigma^{\otimes n}$, with $\rho, \sigma \in \mathcal{D}(\mathcal{H})$ and $\mathcal{H}_{n}=\mathcal{H}^{\otimes n}$. For such states, from the definitions (2.4) and 2.5) of the quantum relative entropy and the quantum information variance it follows that

$$
D\left(\rho_{n} \| \sigma_{n}\right)=n D(\rho \| \sigma) \text { and } \quad V\left(\rho_{n} \| \sigma_{n}\right)=n V(\rho \| \sigma) .
$$

Hence, choosing $w_{n}=n$ in Equation (3.1), we obtain

$$
d(\hat{\rho}, \hat{\sigma})=D(\rho \| \sigma) \quad v(\hat{\rho}, \hat{\sigma})=V(\rho \| \sigma) .
$$

Moreover, $\Psi_{1-z}\left(\rho_{n} \mid \sigma_{n}\right)=n \Psi_{1-z}(\rho \mid \sigma)$.

From the above it follows that 1 is satisfied. Hence, in this case Theorem 1 holds, and more precisely Corollary 1 holds. This result was obtained independently by Tomamichel and Hayashi [44] and Li [28, employing the Central Limit Theorem (CLT). The fact that it follows from our analysis is not surprising, since for i.i.d. states Bryc's theorem reduces to the CLT. In addition, in the i.i.d. case we have the more powerful Berry-Esseen theorem (see e.g. [13]) which can be used to obtain information about the behaviour of the third order term in the expansion of $-\log \beta_{n}(\varepsilon)$, as stated in the following theorem.

Theorem 3. Fix $\varepsilon \in(0,1)$. Then in the case in which the states $\rho_{n}$ and $\sigma_{n}$ in the sequences $\hat{\rho}$ and $\hat{\sigma}$ are i.i.d. states on the sequence of finite dimensional Hilbert spaces $\mathcal{H}^{\otimes n}$, i.e. $\rho_{n}=\rho^{\otimes n}$ and $\sigma_{n}=\sigma^{\otimes n}$, we have

$$
-\log \beta_{n}(\varepsilon)=n D(\rho \| \sigma)+\sqrt{n V(\rho \| \sigma)} \Phi^{-1}(\varepsilon)+\mathcal{O}(\log n) .
$$

Proof. We first prove the bound

$$
-\log \beta_{n}(\varepsilon) \leq n D(\rho \| \sigma)+\sqrt{n V(\rho \| \sigma)} \Phi^{-1}(\varepsilon)+\mathcal{O}(\log n)
$$

Following the proof of Equation (3.3) of Theorem 1 up to Equation (3.16) (with the choice $\left.w_{n}=n\right)$, we infer that for any test $T_{n}$ such that $\operatorname{Tr}\left(\left(\mathbb{I}_{n}-T_{n}\right) \rho_{n}\right) \leq \varepsilon$, for any $\theta_{n}, v_{n} \in \mathbb{R}$

$$
\operatorname{Tr}\left(T_{n} \sigma_{n}\right) \geq \mathrm{e}^{-\theta_{n}}\left(\frac{\mathbb{P}\left(X_{n} \leq v_{n}\right)}{1+\mathrm{e}^{v_{n}-\theta_{n}}}-\varepsilon\right)
$$

where $X_{n} \sim \mu_{\sigma_{n} \mid \rho_{n}}$ is a sum of $n$ i.i.d. random variables of law $\mu_{\sigma \mid \rho}$ (compute e.g. its characteristic function). By the Berry-Esseen theorem, for any real $a, \mathbb{P}\left(\frac{X_{n}-n D(\rho \| \sigma)}{\sqrt{n V(\rho \| \sigma)}} \leq a\right)-$ $\Phi(a)=\mathcal{O}\left(n^{-1 / 2}\right)$. Choosing $v_{n}=n D(\rho \| \sigma)+\sqrt{n V(\rho \| \sigma)} \Phi^{-1}(\varepsilon)$, in Equation 4.2), gives

$$
\operatorname{Tr}\left(T_{n} \sigma_{n}\right) \geq \mathrm{e}^{-\theta_{n}}\left(\frac{\varepsilon+\mathcal{O}\left(n^{-1 / 2}\right)}{1+\mathrm{e}^{v_{n}-\theta_{n}}}-\varepsilon\right)
$$

Further, choosing $\theta_{n}=v_{n}+\log \sqrt{n}$, we obtain

$$
\operatorname{Tr}\left(T_{n} \sigma_{n}\right) \geq \mathrm{e}^{-n D(\rho \| \sigma)-\sqrt{n V(\rho \| \sigma)} \Phi^{-1}(\varepsilon)-\log \sqrt{n}}\left(\frac{\mathcal{O}\left(n^{-1 / 2}\right)-\varepsilon n^{-1 / 2}}{1+n^{-1 / 2}}\right),
$$


so that

$$
-\log \operatorname{Tr}\left(\sigma_{n} T_{n}\right) \leq n D(\rho \| \sigma)+\sqrt{n V(\rho \| \sigma)} \Phi^{-1}(\varepsilon)+\mathcal{O}(\log n) .
$$

Next, to prove the opposite inequality, namely,

$$
-\log \operatorname{Tr}\left(\sigma_{n} T_{n}\right) \leq n D(\rho \| \sigma)+\sqrt{n V(\rho \| \sigma)} \Phi^{-1}(\varepsilon)+\mathcal{O}(\log n),
$$

we proceed similarly to the proof of inequality (3.4) of Theorem 1 (with the choice $w_{n}=n$ ), except that we replace $o\left(\sqrt{w_{n}}\right)$ in the expression of $L_{n}$ with $\mathcal{O}(\log n)$.

\subsection{Quantum Spin Systems}

In this section we consider the example of quantum spin systems on the lattice $\Lambda=\mathbb{Z}^{d}$ (all results below hold for any lattice of finite degree). Here we closely follow the discussion and notation of [24]. Let $\mathcal{X}$ denote the set of all finite subsets of $\Lambda$, and $\mathcal{X}_{0} \subset \mathcal{X}$ the set of subsets of $\Lambda$ containing 0 . At each lattice site $x \in \Lambda$, there is a particle with a finite number of internal (or spin) degrees of freedom, and we assume that each particle has an identical state space $\mathfrak{h}$. For bookkeeping purposes we denote by $\mathfrak{h}_{x}$ the copy of $\mathfrak{h}$ associated with the particle at site $x$. For $X \in \mathcal{X}$ we let $\mathfrak{h}_{X}=\bigotimes_{x \in X} \mathfrak{h}_{x}$ and for $X_{1}, X_{2} \in \mathcal{X}$ with $X_{1} \subset X_{2}$ we identify $\mathfrak{h}_{X_{1}}$ with a subspace of $\mathfrak{h}_{X_{2}}$ via the decomposition $\mathfrak{h}_{X_{2}}=\mathfrak{h}_{X_{1}} \otimes \mathfrak{h}_{X_{2} \backslash X_{1}}$.

Consider the algebras $\mathcal{A}_{X}$ of all bounded operators acting on $\mathfrak{h}_{X}, X \in \mathcal{X}$, with the usual operator norm and with Hermitian conjugation as the $*$-involution. The algebras $\mathcal{A}_{X}$ can be considered to be partially nested, i.e.

$$
\mathcal{A}_{X_{1}} \subset \mathcal{A}_{X_{2}} \text { if } X_{1} \subset X_{2},
$$

by identifying each operator $A_{1} \in \mathcal{A}_{X_{1}}$ with the operator $A_{1} \otimes \mathbb{I}_{X_{2} \backslash X_{1}} \in \mathcal{A}_{X_{2}}$, where $\mathbb{I}$ denotes the identity operator. Moreover, the algebras $\mathcal{A}_{X}$ are local, i.e. if $A_{1} \in \mathcal{A}_{X_{1}}$ and $A_{2} \in \mathcal{A}_{X_{2}}$, and $X_{1} \cap X_{2}=\emptyset$, then $A_{1} A_{2}=A_{2} A_{1}$. The norm closure of $\bigcup_{X \nearrow \mathbb{Z}^{d}} \mathcal{A}_{X}$ defines an algebra which we denote by $\mathcal{A}$. It is the quasilocal $\mathrm{C}^{*}$-algebra of observables associated with the infinite lattice $\mathbb{Z}^{d}$. All local algebras $\mathcal{A}_{X}$ are subalgebras of $\mathcal{A}$, and we denote by $\|A\|$ the norm of any operator $A \in \mathcal{A}$.

For any $X$ in $\mathcal{X}$ we denote by $|X|$ its cardinality, by $\operatorname{diam}(X)$ its diameter

$$
\operatorname{diam}(X):=\max \left\{|x-y|_{\mathbb{Z}^{d}} \text { s.t. } x, y \in X\right\},
$$

where $|x-y|_{\mathbb{Z}^{d}}:=\sum_{i=1}^{d}\left|x_{i}-y_{i}\right|$ denotes the "Manhattan distance" between the two sites $x, y \in \mathbb{Z}^{d}$; here $x=\left(x_{1}, \ldots, x_{d}\right)$ and $y=\left(y_{1}, \ldots, y_{d}\right)$. For $a \in \Lambda$ we define a map $T_{a}$ as the identity $\mathfrak{h}_{x} \rightarrow \mathfrak{h}_{x+a}$ which we extend as a map $\mathfrak{h}_{X} \rightarrow \mathfrak{h}_{X+a}$ where $X+a=\{x+a \mid x \in X\}$. The group of space translations $\mathbf{T}_{\mathbb{Z}^{d}}$ acts as a $*$-automorphism group $\left\{T_{a}: a \in \mathbb{Z}^{d}\right\}$ on $\mathcal{A}$, and for any $X \subset \mathbb{Z}^{d}$

$$
\mathcal{A}_{X+a}=T_{a} \mathcal{A}_{X} T_{-a} .
$$

An interaction of a quantum spin system is a function $\Phi$ from finite, nonempty subsets $X$ of $\mathbb{Z}^{d}$, to self-adjoint observables $\Phi_{X} \in \mathcal{A}_{X}$. The interactions are said to be translation invariant if

$$
T_{a} \Phi_{X} T_{-a}=\Phi_{X+a} \quad \text { for all } a \in \mathbb{Z}^{d}, X \subset \mathbb{Z}^{d}
$$


The following quantity will be relevant for us:

$$
\|\Phi\|=\sum_{X \in \mathcal{X}_{0}}\left\|\Phi_{X}\right\|
$$

We say that the interaction has finite range if there exists an $R>0$ such that $\operatorname{diam} X \geq R$ implies $\Phi(X)=0$; the smallest such $R$ is called the range of $\Phi$. Notice that an interaction $\Phi$ with finite range has finite $\|\Phi\|$. For any $X \in \mathcal{X}$ we associate to an interaction $\Phi$ the so-called interaction Hamiltonian on $X$ : for two fixed interactions $\Phi$ and $\Psi$ we define the following Hamiltonians:

$$
H_{X}^{\Phi}=\sum_{Y \subset X} \Phi_{Y} \quad \text { and } \quad H_{X}^{\Psi}=\sum_{Y \subset X} \Psi_{Y}
$$

We are interested in finite-volume Gibbs states associated with $\Phi$ and $\Psi$ respectively. More precisely, for every $n \in \mathbb{N}$ we let $\Lambda_{n}=\{-n, \ldots,+n\}^{d}$ and define two Gibbs states on $\mathcal{H}_{n}:=\mathfrak{h}_{\Lambda_{n}}$ by density matrices

$$
\rho_{n}=\frac{\mathrm{e}^{-\beta_{1} H_{\Lambda_{n}}^{\Phi}}}{\operatorname{Tr}\left(\mathrm{e}^{-\beta_{1} H_{\Lambda_{n}}^{\Phi}}\right)} \quad \text { and } \quad \sigma_{n}=\frac{\mathrm{e}^{-\beta_{2} H_{\Lambda_{n}}^{\Psi}}}{\operatorname{Tr}\left(\mathrm{e}^{-\beta_{2} H_{\Lambda_{n}}^{\Psi}}\right)}
$$

where $\beta_{1}, \beta_{2} \in(0, \infty)$ are inverse temperatures (the physical definition would actually require $\beta=\left(k_{B} T\right)^{-1}$ where $T$ is the temperature and $k_{B}$ is Boltzmann's constant; here we set $\left.k_{B}=1\right)$. Using the results of [33] we can prove the following:

Proposition 2. If $\Phi$ and $\Psi$ are translation invariant, finite range interactions, then for high enough temperatures (i.e. for inverse temperatures $\beta_{1}$ and $\beta_{2}$ small enough), the sequence $\left(\rho_{n}, \sigma_{n}\right)_{n \in \mathbb{N}}$ of pairs of finite volume Gibbs states on $\Lambda_{n}=\{-n, \ldots, n\}^{d}$ (defined through Equation 4.5 above), satisfies 1 with respect to the weights $w_{n}=\left|\Lambda_{n}\right|=(2 n+1)^{d}$.

Proof. In the present context, the quantity $E_{n}(z)$ appearing in 1 is given by

$$
\begin{aligned}
E_{n}(z) & :=\Psi_{1-z}\left(\rho_{n} \mid \sigma_{n}\right) \\
& =\log \operatorname{Tr}\left(\mathrm{e}^{-(1-z) \beta_{1} H_{\Lambda_{n}}^{\Phi}} \mathrm{e}^{-z \beta_{2} H_{\Lambda_{n}}^{\Psi}}\right)-(1-z) \log \operatorname{Tr}\left(\mathrm{e}^{-\beta_{1} H_{\Lambda_{n}}^{\Phi}}\right)-z \log \operatorname{Tr}\left(\mathrm{e}^{-\beta_{2} H_{\Lambda_{n}}^{\Psi}}\right) .
\end{aligned}
$$

It is a well-known result (see e.g. [41]) that, under the assumptions of Proposition 2, the quantities

$$
\frac{1}{\left|\Lambda_{n}\right|} \log \operatorname{Tr}\left(\mathrm{e}^{-\beta_{1} H_{\Lambda_{n}}^{\Phi}}\right) \quad \text { and } \quad \frac{1}{\left|\Lambda_{n}\right|} \log \operatorname{Tr}\left(\mathrm{e}^{-\beta_{2} H_{\Lambda_{n}}^{\Psi}}\right)
$$

converge as $n \rightarrow \infty$, the limits being called the pressure associated with the pairs $\left(\beta_{1}, \Phi\right)$ and $\left(\beta_{2}, \Psi\right)$, respectively. Hence, to establish 1 it suffices to prove that there exists an $r>0$ such that the functions

$$
f_{n}(z):=\frac{1}{\left|\Lambda_{n}\right|} \log \operatorname{Tr}\left(\mathrm{e}^{-(1-z) \beta_{1} H_{\Lambda_{n}}^{\Phi}} \mathrm{e}^{-z \beta_{2} H_{\Lambda_{n}}^{\Psi}}\right)
$$

are analytic on $B_{\mathbb{C}}(0, r)$, converge pointwise on the real segment $(-r,+r)$, and admit a uniform (in $n$ and $z$ ) bound. 
Our proof relies on a result of [33], namely Proposition 7.10 of the paper, which in the present context can be stated as Proposition 3 below. Before stating it, however, we need to define certain quantities.

Consider the completely mixed state acting on the Hilbert space $\mathcal{H}_{n}:=\mathfrak{h}_{\Lambda_{n}}$. It is an element of the observable algebra $\mathcal{A}_{\Lambda_{n}}$ and is given by the tensor product (or i.i.d.) density matrix

$$
\tau_{n}:=\bigotimes_{x \in \Lambda_{n}} \frac{I_{x}}{\operatorname{dim} \mathfrak{h}}=\frac{1}{(\operatorname{dim} \mathfrak{h})^{\left|\Lambda_{n}\right|}} \bigotimes_{x \in \Lambda_{n}} I_{x}
$$

where $I_{x} \in \mathcal{A}_{x}$ denotes the identity operator acting on $\mathfrak{h}_{x}$, and $\operatorname{dim} \mathfrak{h}$ is the dimension of $\mathfrak{h}$ (recall $\left.\mathfrak{h}_{x} \simeq \mathfrak{h}\right)$.

Further, define the following quantity, for any $z_{1}, z_{2} \in \mathbb{C}$ :

$$
Z_{\Lambda_{n}}^{z_{1}, z_{2}}:=\operatorname{Tr}\left(\tau_{n} e^{z_{1} \beta_{1} H_{\Lambda_{n}}^{\Phi}} e^{z_{2} \beta_{2} H_{\Lambda_{n}}^{\Psi}}\right) .
$$

Its logarithm is hence given by

$$
\log Z_{\Lambda_{n}}^{z_{1}, z_{2}}=-\left|\Lambda_{n}\right| \log (\operatorname{dim} \mathfrak{h})+\log \operatorname{Tr}\left(e^{z_{1} \beta_{1} H_{\Lambda_{n}}^{\Phi}} e^{z_{2} \beta_{2} H_{\Lambda_{n}}^{\Psi}}\right) .
$$

Note in particular, that for the choice $z_{1}=z-1$ and $z_{2}=-z$, the function $f_{n}(z)$ (defined through Equation (4.7)), whose analyticity and convergence properties we are interested in, can be expressed in terms of the above quantity as follows:

$$
f_{n}(z)=\frac{1}{\left|\Lambda_{n}\right|} \log Z_{\Lambda_{n}}^{z-1,-z}+\log (\operatorname{dim} \mathfrak{h}) .
$$

Hence, we can deduce the desired properties of $f_{n}(z)$ from those of $\log Z_{\Lambda_{n}}^{z-1,-z}$.

The quantity $\log Z_{\Lambda_{n}}^{z_{1}, z_{2}}$ has an expansion of the form (see [33])

$$
\log Z_{\Lambda_{n}}^{z_{1}, z_{2}}=\sum_{C \subseteq \Lambda_{n}} \mathrm{w}^{z_{1}, z_{2}}(C),
$$

where the cluster weights $\mathrm{w}^{z_{1}, z_{2}}(C)$ are also translation-invariant, i.e.

$$
\mathrm{w}^{z_{1}, z_{2}}(C)=\mathrm{w}^{z_{1}, z_{2}}(C+x)
$$

for any $x \in \Lambda, C \in \mathcal{X}$. Such an expansion is called a cluster expansion. Proposition 7.10 of [33] is a statement of analyticity of these cluster weights and the boundedness of the cluster expansion. An immediate simplification can be stated as follows:

Proposition 3. Assume that $\Phi$ and $\Psi$ are translation-invariant interactions, and

$$
\sum_{X \in \mathcal{X}_{0}} e^{2 a|X|}\left(e^{\delta\left(\beta_{1}\left\|\Phi_{X}\right\|+\beta_{2}|| \Psi_{X} \|\right)}-1\right) \leq a,
$$

for some $a, \delta>0$. Then all cluster weights are analytic in $\mathcal{D}:=B_{\mathbb{C}}(0, \delta)^{2}$ and

$$
\sup _{\left(z_{1}, z_{2}\right) \in \mathcal{D}} \sum_{C_{0} \in \mathcal{X}_{0}}\left|\mathrm{w}^{z_{1}, z_{2}}\left(C_{0}\right)\right| \leq a
$$


To employ the above proposition in our proof, we proceed as follows. Denote by $R$ the range of $\Phi$, and let $q=\max \left\{\beta_{1}\|\Phi\|, \beta_{2}\|\Psi\|\right\}$. We fix $a>0$ and $\delta=1+r>1$. The number of sets $X$ that contain the origin 0 , and have diameter at most $R$ is bounded by $2^{N_{R, d}}$ with $N_{R, d}=(2 R+1)^{d}$. A crude upper bound for the left hand side of Equation (4.11) is

$$
2^{N_{R, d}} e^{2 a N_{R, d}}\left(e^{2 \delta q}-1\right) .
$$

Since this upper bound is zero for $q=0$, it remains smaller than $a$ for $q$ small but nonzero. For this value of $q$, 4.11) holds, so that the claim of the proposition holds with $\mathcal{D}=B_{\mathbb{C}}(0,1+r)^{2}$. In particular, setting $z_{1}=z-1$ and $z_{2}=-z$, and using the translation invariance of the interaction $\Phi$ and $\Psi$, we infer that

$$
\sup _{z \in B_{\mathbb{C}}(0, r)} \sum_{C_{0} \in \mathcal{X}_{0}}\left|\mathrm{w}^{z-1,-z}\left(C_{0}\right)\right| \leq a .
$$

Now observe that any $C \in \mathcal{X}$ can be written $x+C_{0}$ with $x \in \Lambda$ and $C_{0} \in \mathcal{X}_{0}$ in exactly $|C|=\left|C_{0}\right|$ distinct ways. Then, using the notation $P_{c}$, which is equal to 1 if the condition $c$ holds and 0 otherwise, we have

$$
\begin{aligned}
\sum_{C \subset \Lambda_{n}} \mathrm{w}^{z-1,-z}(C) & =\sum_{x \in \Lambda_{n}} \sum_{C_{0} \in \mathcal{X}_{0}} P_{x+C_{0} \subseteq \Lambda_{n}} \frac{\mathrm{w}^{z-1,-z}\left(C_{0}\right)}{\left|C_{0}\right|} \\
\left|\Lambda_{n}\right| \sum_{C_{0} \in \mathcal{X}_{0}} \frac{\mathrm{w}^{z-1,-z}\left(C_{0}\right)}{\left|C_{0}\right|} & =\sum_{x \in \Lambda_{n}} \sum_{C_{0} \in \mathcal{X}_{0}} \frac{\mathrm{w}^{z-1,-z}\left(C_{0}\right)}{\left|C_{0}\right|}
\end{aligned}
$$

so that by 4.9 ,

$$
\sum_{C_{0} \in \mathcal{X}_{0}} \frac{\mathrm{w}^{z-1,-z}\left(C_{0}\right)}{\left|C_{0}\right|}-\frac{1}{\left|\Lambda_{n}\right|} \sum_{C \subseteq \Lambda_{n}} \mathrm{w}^{z-1,-z}(C)=\frac{1}{\left|\Lambda_{n}\right|} \sum_{x \in \Lambda_{n}} \sum_{C_{0} \in \mathcal{X}_{0}} P_{x+C_{0} \nsubseteq \Lambda_{n}} \frac{\mathrm{w}^{z-1,-z}\left(C_{0}\right)}{\left|C_{0}\right|} .
$$

By Equation (4.13), one has

$$
\left|\sum_{C_{0} \in \mathcal{X}_{0}} \frac{\mathrm{w}^{z-1,-z}\left(C_{0}\right)}{\left|C_{0}\right|}\right| \leq \sum_{C_{0} \in \mathcal{X}_{0}} \frac{\left|\mathrm{w}^{z-1,-z}\left(C_{0}\right)\right|}{\left|C_{0}\right|} \leq \sum_{C_{0} \in \mathcal{X}_{0}}\left|\mathrm{w}^{z-1,-z}\left(C_{0}\right)\right| \leq a
$$

for any $z \in B_{\mathbb{C}}(0, r)$. Now, by Equations 4.9$)$ and (4.10), 1 will follow for this model if we can prove

$$
\lim _{n \rightarrow \infty} \sup _{z \in B_{\mathbb{C}}(0, r)}\left|\frac{1}{\left|\Lambda_{n}\right|} \sum_{x \in \Lambda_{n}} \sum_{C_{0} \in \mathcal{X}_{0}} P_{x+C_{0} \nsubseteq \Lambda_{n}} \frac{\mathrm{w}^{z-1,-z}\left(C_{0}\right)}{\left|C_{0}\right|}\right|=0 .
$$

Fix $z \in B_{\mathbb{C}}(0, r)$. From (4.13), for any $\varepsilon>0$, there exists a finite set $\Sigma \subset \mathcal{X}_{0}$ such that

$$
\sum_{C_{0} \in \mathcal{X}_{0} \backslash \Sigma}\left|\mathrm{w}^{z-1,-z}\left(C_{0}\right)\right|<\varepsilon
$$

Denote by $S=\max \left\{\operatorname{diam} C_{0} \mid C_{0} \in \Sigma\right\}$, and let

$$
\Lambda_{n}^{\Sigma}=\left\{x \in \Lambda_{n} \mid x+C_{0} \subset \Lambda_{n} \forall C_{0} \in \Sigma\right\} .
$$


Obviously for $n>S$ one has $\Lambda_{n-S} \subset \Lambda_{n}^{\Sigma}$, and

$$
\begin{aligned}
\left|\sum_{x \in \Lambda_{n}} \sum_{C_{0} \in \mathcal{X}_{0}} P_{x+C_{0} \nsubseteq \Lambda_{n}} \frac{\mathrm{w}^{z-1,-z}\left(C_{0}\right)}{\left|C_{0}\right|}\right| \leq & \sum_{x \in \Lambda_{n}} \sum_{C_{0} \in \mathcal{X}_{0}} P_{x+C_{0} \nsubseteq \Lambda_{n}} \frac{\left|\mathrm{w}^{z-1,-z}\left(C_{0}\right)\right|}{\left|C_{0}\right|} \\
\leq & \sum_{x \in \Lambda_{n}^{\Sigma}} \sum_{C_{0} \in \mathcal{X}_{0}} P_{x+C_{0} \nsubseteq \Lambda_{n}} \frac{\left|\mathrm{w}^{z-1,-z}\left(C_{0}\right)\right|}{\left|C_{0}\right|} \\
& +\sum_{x \in \Lambda_{n} \backslash \Lambda_{n}^{\Sigma}} \sum_{C_{0} \in \mathcal{X}_{0}} P_{x+C_{0} \nsubseteq \Lambda_{n}} \frac{\left|\mathrm{w}^{z-1,-z}\left(C_{0}\right)\right|}{\left|C_{0}\right|} .
\end{aligned}
$$

By definition of $\Lambda_{n}^{\Sigma}$ one has

$$
\sum_{x \in \Lambda_{n}^{\Sigma}} \sum_{C_{0} \in \mathcal{X}_{0}} P_{x+C_{0} \nsubseteq \Lambda_{n}} \frac{\left|\mathrm{w}^{z-1,-z}\left(C_{0}\right)\right|}{\left|C_{0}\right|} \leq\left|\Lambda_{n}^{\Sigma}\right| \sum_{C_{0} \in \mathcal{X}_{0} \backslash \Sigma}\left|\mathrm{w}^{z-1,-z}\left(C_{0}\right)\right|<\varepsilon\left|\Lambda_{n}^{\Sigma}\right| .
$$

On the other hand,

$$
\sum_{x \in \Lambda_{n} \backslash \Lambda_{n}^{\Sigma}} \sum_{C_{0} \in \mathcal{X}_{0}} P_{x+C_{0} \nsubseteq \Lambda_{n}} \frac{\left|\mathrm{w}^{z-1,-z}\left(C_{0}\right)\right|}{\left|C_{0}\right|} \leq\left|\Lambda_{n} \backslash \Lambda_{n}^{\Sigma}\right| \sum_{C_{0} \in \mathcal{X}_{0}}\left|\mathrm{w}^{z-1,-z}\left(C_{0}\right)\right| \leq a\left|\Lambda_{n} \backslash \Lambda_{n}^{\Sigma}\right| .
$$

This implies immediately

$$
\sup _{z \in B_{\mathbb{C}}(0, r)}\left|\frac{1}{\left|\Lambda_{n}\right|} \sum_{x \in \Lambda_{n}} \sum_{C_{0} \in \mathcal{X}_{0}} P_{x+C_{0} \nsubseteq \Lambda_{n}} \frac{\mathrm{w}^{z-1,-z}\left(C_{0}\right)}{\left|C_{0}\right|}\right| \leq \varepsilon+a\left(1-\left(\frac{2(n-S)+1}{2 n+1}\right)^{d}\right),
$$

where we use the fact that $\left|\Lambda_{n-S}\right| \leq\left|\Lambda_{n}^{\Sigma}\right|$. Since $\varepsilon$ is arbitrary, this implies Equation 4.14 and therefore 1 .

\subsection{Free fermions on a lattice}

In this section we consider quasi-free states of a fermionic lattice gas. We closely follow the treatment of [31]. This is given, for example, by states of non-interacting electrons on the lattice $\mathbb{Z}^{d}$, which are allowed to hop from site to site. Since the electrons are fermions, they are subject to Fermi-Dirac statistics and hence they have to obey the Pauli exclusion principle. The prefix "quasi" in "quasi-free" can be heuristically understood to arise from the fact that, even though the electrons do not interact among themselves, their movement on the lattice is constrained by the Fermi-Dirac statistics.

Let us give a brief description of the setup. Let us start with the one-particle Hilbert space $\mathfrak{h}:=\ell^{2}\left(\mathbb{Z}^{d}\right)$. It represents a single particle which is confined to the lattice $\Lambda:=\mathbb{Z}^{d}$. To incorporate the statistics of the particle, we consider the Fock space $\mathcal{F}(\mathfrak{h}):=\oplus_{n \in \mathbb{N}} \wedge^{n} \mathfrak{h}$, with the convention $\wedge^{0}=\mathbb{C}$. Here we use the notation

$$
x_{1} \wedge x_{2} \ldots \wedge x_{n}=\frac{1}{\sqrt{n !}} \sum_{\sigma \in S_{n}} \operatorname{sgn}(\sigma) x_{\sigma(1)} \otimes x_{\sigma(2)} \ldots \otimes x_{\sigma(n)}
$$


where the summation runs over all permutations of $n$ elements and $\operatorname{sgn}(\sigma)$ denotes the sign of the permutation $\sigma$. The algebra of observables is the $\mathrm{C}^{*}$-algebra generated by the creation and annihilation operators on the Fock space, obeying the canonical anticommutation relations (CAR). More precisely, for each $x \in \mathfrak{h}$, the creation operator $a^{*}(x)$ is the unique bounded linear extension $a^{*}(x): \mathcal{F}(\mathfrak{h}) \mapsto \mathcal{F}(\mathfrak{h})$ of

$$
a^{*}(y): x_{1} \wedge \ldots \wedge x_{n} \mapsto y \wedge x_{1} \wedge \ldots \wedge x_{n}, \quad x_{1}, \ldots, x_{n} \in \mathfrak{h}, n \in \mathbb{N},
$$

and the corresponding annihilation operator is its adjoint $a(x):=\left(a^{*}(x)\right)^{*}$. Creation and annihilation operators satisfy the canonical anticommutation relations (CAR):

$$
a(x) a(y)+a(y) a(x)=0, \quad a(x) a^{*}(y)+a^{*}(y) a(x)=\langle x, y\rangle \mathbb{I}
$$

The $\mathrm{C}^{*}$-algebra generated by $\{a(x): x \in \mathfrak{h}\}$ is called the algebra of the canonial anticommutation relations, and is denoted by $\operatorname{CAR}(\mathfrak{h})$. The number operator $N$ is defined in terms of the creation and annihilation operators as follows: $N=\sum_{k} a_{k}^{*} a_{k}$ where $a_{k}=a\left(e_{k}\right)$ for some orthonormal basis $\left\{e_{k}\right\}$ of $\mathfrak{h}$. The algebra $\operatorname{CAR}(\mathfrak{h})$ is infinite-dimensional. However, the objects associated with $\mathfrak{h}_{n}=\ell^{2}\left(\Lambda_{n}\right)$, where $\Lambda_{n}$ is a finite subset of the lattice $\mathbb{Z}^{d}$, are finite-dimensional.

Let us denote the standard basis in $\ell^{2}\left(\mathbb{Z}^{d}\right)$ by $\left\{e_{\mathbf{i}}: \mathbf{i} \in \mathbb{Z}^{d}\right\}$. Then shift operators are defined as the unique linear extensions of $T_{\mathbf{j}}: e_{\mathbf{i}} \rightarrow e_{\mathbf{i}+\mathbf{j}}, \mathbf{i} \in \mathbb{Z}^{d}, \forall \mathbf{j} \in \mathbb{Z}^{d}$. An operator which commutes with all the unitaries $T_{\mathbf{j}}$ is said to be shift-invariant. Shift-invariant operators on $l^{2}\left(\mathbb{Z}^{d}\right)$ commute with each other. Moreover, defining the Fourier transformation

$$
\mathfrak{F}: \ell^{2}\left(\mathbb{Z}^{d}\right) \rightarrow L^{2}\left([0,2 \pi)^{d}\right) ; \quad \mathfrak{F} e_{\mathbf{k}}:=\varphi_{\mathbf{k}}(\mathbf{x}), \quad \text { where } \varphi_{\mathbf{k}}(\mathbf{x})=e^{i\langle\mathbf{k}, \mathbf{x}\rangle}, \mathbf{x} \in[0,2 \pi)^{d}, \mathbf{k} \in \mathbb{Z}^{d},
$$

where $\langle\mathbf{k}, \mathbf{x}\rangle:=\sum_{i=1}^{d} k_{i} x_{i}$, every shift-invariant operator $Q$ can be expressed in the form $Q=\mathfrak{F}^{-1} M_{\hat{q}} \mathfrak{F}$, where $M_{\hat{q}}$ denotes the multiplication operator by a bounded measurable function $\hat{q}$ on $[0,2 \pi)^{d}$. The operator of $Q$ in the basis $\left\{e_{\mathbf{i}}: \mathbf{i} \in \mathbb{Z}^{d}\right\}$ is a Toeplitz operator, and its elements are given by

$$
Q_{\mathbf{j}, \mathbf{k}} \equiv\left\langle e_{\mathbf{j}}, Q e_{\mathbf{k}}\right\rangle=\frac{1}{(2 \pi)^{d}} \int e^{-i\langle\mathbf{j}-\mathbf{k}, \mathbf{x}\rangle} \hat{q}(\mathbf{x}) d \mathbf{x}
$$

Let $H \in \mathcal{B}(\mathfrak{h})$ denote the one-particle Hamiltonian of a system of free fermions on the lattice $\Lambda$. Then an example of a quasi-free state of a fermionic lattice gas is its Gibbs state, which for an inverse temperature $\beta$, is given by the density matrix $\rho_{\beta}:=e^{-\beta \hat{H}} / \operatorname{Tr}\left(e^{-\beta \hat{H}}\right)$, where $\hat{H}=\mathrm{d} \Gamma(H)$ denotes the (differential) second quantization of $H$, acting on the Fock space $\mathcal{F}(\mathfrak{h})$ (see [10] or Merkli's notes in [3]). Consider, in particular, examples in which the Hamiltonian is the second quantization of a shift-invariant operator, implying that the total number of fermions is conserved. The Gibbs state can be expressed as a linear form on $\operatorname{CAR}(\mathfrak{h})$ as follows

$$
\begin{aligned}
\omega_{Q}\left(a^{*}\left(x_{1}\right) \ldots a^{*}\left(x_{n}\right) a\left(y_{m}\right) \ldots a\left(y_{1}\right)\right) & :=\operatorname{Tr}\left(\rho_{\beta} a^{*}\left(x_{1}\right) \ldots a^{*}\left(x_{n}\right) a\left(y_{m}\right) \ldots a\left(y_{1}\right)\right) \\
& =\delta_{m n} \operatorname{det}\left(\left\langle y_{i}, Q x_{j}\right\rangle\right)_{i, j},
\end{aligned}
$$

where $Q=\mathrm{e}^{-\beta H}\left(1+\mathrm{e}^{-\beta H}\right)^{-1} \in \mathcal{B}(\mathfrak{h})$, where $H$ denotes the one-particle Hamiltonian. The state $\omega_{Q}$ is in then called a quasi-free state with symbol $Q$. It is shift-invariant, since the underlying Hamiltonian is chosen to be shift-invariant. 
For our quantum hypothesis testing problem we proceed as follows. Consider a sequence of finite subsets $\Lambda_{n}:=\{0, \ldots, n-1\}^{d} \subset \mathbb{Z}^{d}$ and the subspaces $\mathfrak{h}_{n}=\ell^{2}\left(\Lambda_{n}\right)$ which we can view as subspaces of $\mathfrak{h}$. Let $\omega_{Q}$ and $\omega_{R}$ be two shift-invariant Gibbs states with symbols $Q$ and $R$ respectively, such that $\delta<Q, R<1-\delta$ for some $\delta \in(0,1 / 2)$. By the discussion above, the operators $\mathfrak{F} Q \mathfrak{F}^{-1}$ and $\mathfrak{F} R \mathfrak{F}^{-1}$ on $L^{2}\left([0,2 \pi)^{d}\right)$ are multiplication operators by bounded measurable functions $\hat{q}, \hat{r}:[0,2 \pi)^{d} \rightarrow[0,1]$ with essential range in $[\delta, 1-\delta]$. This condition ensures that the "local restrictions" of $\omega_{Q}, \omega_{R}$ which we now define, are faithful. Let $Q_{n}=$ $P_{n} Q P_{n}, R_{n}=P_{n} R P_{n}$, where $P_{n}$ denotes the orthogonal projection operator on $\mathfrak{h}_{n}$. Then we choose the sequence of states $\left(\rho_{n}, \sigma_{n}\right)_{n \in \mathbb{N}}$ of our hypothesis testing problem to be given by density matrices corresponding to states $\omega_{Q_{n}}$ and $\omega_{R_{n}}$ on $\operatorname{CAR}\left(\mathfrak{h}_{n}\right)$, associated with the symbols $Q_{n}, R_{n}$ respectively through Equation (4.15). That is, the null-hypothesis in this case is that the true state of the infinite system is $\omega_{Q}$, while the alternative hypothesis is that it is $\omega_{R}$, and we make measurements on local subsystems to decide between these two options. By Lemma 3 in [12], the density matrices $\rho_{n}, \sigma_{n}$ associated with $\omega_{Q_{n}}$ and $\omega_{R_{n}}$ are

$$
\rho_{n}=\operatorname{det}\left(\mathbb{I}-Q_{n}\right) \bigoplus_{k=0}^{\operatorname{dim} \mathfrak{h}_{n}} \bigwedge^{k} \frac{Q_{n}}{\mathbb{I}-Q_{n}} \quad \sigma_{n}=\operatorname{det}\left(\mathbb{I}-R_{n}\right) \bigoplus_{k=0}^{\operatorname{dim} \mathfrak{h}_{n}} \bigwedge^{k} \frac{R_{n}}{\mathbb{I}-R_{n}}
$$

where for any operator $A$ on $\mathfrak{h}, \wedge^{k} A$ denotes the restriction of $A^{\otimes k}$ to the antisymmetric subspace $\wedge^{k} \mathfrak{h}$ of $\mathfrak{h}^{\otimes k}$. As shown in [31], it follows from Equation 4.16) that for any $s \in \mathbb{R}$

$$
\Psi_{s}\left(\rho_{n} \mid \sigma_{n}\right)=\operatorname{Tr} \log \left(\mathbb{I}_{n}-Q_{n}\right)^{s}+\operatorname{Tr} \log \left(\mathbb{I}-R_{n}\right)^{1-s}+\operatorname{Tr} \log \left(\mathbb{I}_{n}+W_{n}^{(s)}\right),
$$

where

$$
W_{n}^{(s)}=\left(\frac{Q_{n}}{\mathbb{I}_{n}-Q_{n}}\right)^{\frac{s}{2}}\left(\frac{R_{n}}{\mathbb{I}_{n}-R_{n}}\right)^{1-s}\left(\frac{Q_{n}}{\mathbb{I}_{n}-Q_{n}}\right)^{\frac{s}{2}} .
$$

and

$$
\begin{aligned}
D\left(\rho_{n} \| \sigma_{n}\right)= & \left.\frac{\mathrm{d}}{\mathrm{d} s} \Psi_{s}\left(\rho_{n} \mid \sigma_{n}\right)\right|_{s=1} \\
= & \operatorname{Tr}\left(Q_{n} \log Q_{n}\right)+\operatorname{Tr}\left(\left(\mathbb{I}_{n}-Q_{n}\right) \log \left(\mathbb{I}_{n}-Q_{n}\right)\right) \\
& \quad-\operatorname{Tr}\left(Q_{n} \log R_{n}\right)-\operatorname{Tr}\left(\left(\mathbb{I}_{n}-Q_{n}\right) \log \left(\mathbb{I}_{n}-R_{n}\right)\right) .
\end{aligned}
$$

Proposition 4. For the sequence $\left(\rho_{n}, \sigma_{n}\right)_{n \in \mathbb{N}}$ defined above, we have

$$
\begin{aligned}
d(\hat{\rho}, \hat{\sigma}) & :=\lim _{n \rightarrow \infty} \frac{1}{n^{d}} D\left(\rho_{n} \| \sigma_{n}\right) \\
& =\frac{1}{(2 \pi)^{d}} \int_{[0,2 \pi)^{d}} \hat{q}(\boldsymbol{x}) \log \frac{\hat{q}(\boldsymbol{x})}{\hat{r}(\boldsymbol{x})}+(1-\hat{q}(\boldsymbol{x})) \log \frac{1-\hat{q}(\boldsymbol{x})}{1-\hat{r}(\boldsymbol{x})} \mathrm{d} \boldsymbol{x} .
\end{aligned}
$$

and for any $s \in \mathbb{R}$

$$
\lim _{n \rightarrow \infty} \frac{1}{n^{d}} \Psi_{s}\left(\rho_{n} \mid \sigma_{n}\right)=\frac{1}{(2 \pi)^{d}} \int_{[0,2 \pi)^{d}} \log \left(\hat{q}^{s}(\boldsymbol{x}) \hat{r}^{1-s}(\boldsymbol{x})+(1-\hat{q}(\boldsymbol{x}))^{s}(1-\hat{r}(\boldsymbol{x}))^{1-s}\right) \mathrm{d} \boldsymbol{x} .
$$

Moreover the sequence $\left(\rho_{n}, \sigma_{n}\right)_{n \in \mathbb{N}}$ satisfies 1 with respect to the weights $w_{n}=\left|\Lambda_{n}\right|$. Assuming moreover that $Q$ satisfies

$$
\sum_{k \in \mathbb{Z}^{d}}|\boldsymbol{k}|^{d}\left|\left\langle e_{\boldsymbol{k}}, Q e_{\mathbf{0}}\right\rangle\right|<\infty
$$


then

$$
D\left(\rho_{n} \| \sigma_{n}\right)-n^{d} d(\hat{\rho}, \hat{\sigma})=\mathcal{O}\left(n^{d-1}\right) .
$$

Remark 5. This proves that whenever Equation (4.22) is satisfied, then the assumptions of Corollary 1 hold for $d=1$. In the general case, denote $C$ such that $D\left(\rho_{n} \| \sigma_{n}\right)=n^{2} d(\hat{\rho}, \hat{\sigma})+$ $n C+O(1)$, then for $d=2$, the second order of $-\log \beta_{n}(\varepsilon)$ is essentially given by $n\left(t_{2}^{*}(\varepsilon)+C\right)$ and for $d \geq 3$ it is given by $n^{d-1} C$.

Remark 6. The stronger assumption of Equation 4.22 holds in particular if the two states considered here are Gibbs states at different temperatures, under the condition that for some $\beta>0$,

$$
\sum_{\mathbf{k} \in \mathbb{Z}^{d}}|\mathbf{k}|^{d}\left|\left\langle e_{\mathbf{k}}, \frac{\mathrm{e}^{-\beta H}}{1+\mathrm{e}^{-\beta H}} e_{\mathbf{0}}\right\rangle\right|<+\infty,
$$

This proves that for such systems we are in the case where Corollary 1 holds.

Proof. Equation (4.20) and Equation (4.21) (which is point 2 of 1) were proved in 31. We now prove that the first and third parts of 1 hold as well. For any $n$, let $A_{n}=\frac{Q_{n}}{\mathbb{I}_{n}-Q_{n}}$ and $B_{n}=\frac{R_{n}}{\mathbb{I}_{n}-R_{n}}$. We have $M^{-1} \leq A_{n} \leq M$, and $M^{-1} \leq B_{n} \leq M$ for $M=(1-\delta) \delta^{-1}$. The operators $A_{n}$ and $B_{n}$ are self-adjoint and we can therefore define their complex powers. Define $W_{n}^{(1-z)}=A_{n}^{\frac{1-z}{2}} B_{n}^{z} A_{n}^{\frac{1-z}{2}}$. As $A_{n}^{z}=A_{n}^{s} A_{n}^{i t}$, we have for $r \in(0,1)$ and any $z=s+i t$ in $B_{\mathbb{C}}(0, r)$,

$$
\max \left(\left\|A_{n}^{z}\right\|,\left\|B_{n}^{z}\right\|\right) \leq M^{|s|} \text { so that }\left\|W_{n}^{(1-z)}\right\| \leq M^{1+2 r} .
$$

As any differentiable function is locally Lipschitz, with Lipschitz constant given by the supremum norm of its derivative over each bounded interval, we also obtain the following bound

$$
\left\|A_{n}^{s}-A_{n}^{s+i t}\right\|=\left\|A_{n}^{s+i 0}-A_{n}^{s+i t}\right\| \leq \sup _{0 \leq u \leq t}\left\|\left(\log A_{n}\right) A_{n}^{s+i u}\right\||t| \leq r M^{|s|} \log M .
$$

Proceeding in the same manner with $B_{n}$, we conclude that there exists a constant $C:=\log M$ such that

$$
\max \left(\left\|A_{n}^{s}-A_{n}^{s+i t}\right\|,\left\|B_{n}^{s}-B_{n}^{s+i t}\right\|\right) \leq C M^{|s|} r \quad \text { if }|t|<r .
$$

Using Equation (4.24, Equation (4.26) and the triangle inequality, we obtain

$$
\begin{aligned}
\left\|W_{n}^{(1-z)}-W_{n}^{(1-s)}\right\| & =\left\|A_{n}^{\frac{1-z}{2}} B_{n}^{z} A_{n}^{\frac{1-z}{2}}-A_{n}^{\frac{1-s}{2}} B_{n}^{s} A_{n}^{\frac{1-s}{2}}\right\| \\
& \leq\left\|A_{n}^{1 / 2}\right\|^{2}\left\|A_{n}^{-z / 2}\left(B_{n}^{z}-B_{n}^{s}\right) A_{n}^{-z / 2}+A_{n}^{-z / 2} B_{n}^{s} A_{n}^{-z / 2}-A_{n}^{-s / 2} B_{n}^{s} A_{n}^{-s / 2}\right\| \\
& \leq M^{1+2|s|} C r+M\left\|\left(A_{n}^{-z / 2}-A_{n}^{-s / 2}\right) B_{n}^{s} A_{n}^{-z / 2}+A_{n}^{-s / 2} B_{n}^{s}\left(A_{n}^{-z / 2}-A_{n}^{-s / 2}\right)\right\| \\
& \leq 3 M^{1+2|s|} C r \leq 3 M^{1+2 r} C r,
\end{aligned}
$$

Theorem VI.3.3 from [5] implies that

$$
\max _{\lambda \in \operatorname{sp} W_{n}^{(z)}} \min _{\mu \in \operatorname{sp} W_{n}^{(s)}}|\lambda-\mu| \leq 3 C M^{1+2 r} r .
$$


Since $\operatorname{sp} W_{n}^{(1-s)} \subset\left[M^{-(1+2 r)}, M^{1+2 r}\right]$, Equation 4.28) implies that, for $r$ small enough, the spectrum of $W_{n}^{(1-z)}$ remains in the half plane $\left\{z^{\prime} \in \mathbb{C} \mid \operatorname{Re} z^{\prime}>0\right\}$ for any $n$ and $z \in B_{\mathbb{C}}(0, r)$. Then considering the principal branch of the complex logarithm (which we simply denote by $\log$ ), we can define $\operatorname{Tr} \log \left(\mathbb{I}_{n}+W_{n}^{(1-z)}\right)$ by Dunford-Taylor functional calculus (see [26]) for any $z \in B_{\mathbb{C}}(0, r)$, and it will be an analytic function of $z$. Then expression (4.17) gives us an analytic extension of $\frac{1}{n^{d}} \Psi_{1-z}\left(\rho_{n} \mid \sigma_{n}\right)$ to $z \in B_{\mathbb{C}}(0, r)$, and a uniform bound is provided by

$$
\begin{gathered}
\max \left(\left|\operatorname{Tr} \log \left(\mathbb{I}_{n}-Q_{n}\right)\right|,\left|\operatorname{Tr} \log \left(\mathbb{I}_{n}-R_{n}\right)\right|\right) \leq n^{d} \log (1+M), \\
\left|\operatorname{Tr} \log \left(\mathbb{I}_{n}+W_{n}^{(1-z)}\right)\right| \leq n^{d} \log \left(1+M^{1+2 r}\right) .
\end{gathered}
$$

Therefore, points 1 and 3 of 1 hold.

We now turn to the estimate of $D\left(\rho_{n} \| \sigma_{n}\right)-n^{d} d(\hat{\rho}, \hat{\sigma})$. Using the fact that $Q_{n}$ and $R_{n}$ are truncated Toeplitz operators, as well as $\left\langle\mathrm{e}_{\mathbf{i}}, Q_{n} e_{\mathbf{j}}\right\rangle=\left\langle\mathrm{e}_{\mathbf{i}}, Q e_{\mathbf{j}}\right\rangle$ for any $\mathbf{i}, \mathbf{j} \in\{0, \ldots, n-1\}^{d}$, we have

$$
\begin{aligned}
\operatorname{Tr}\left(Q_{n} \log R_{n}\right)= & \sum_{\mathbf{i} \in\{0, \ldots, n-1\}^{d}}\left\langle e_{\mathbf{i}}, Q_{n} \log R_{n} e_{\mathbf{i}}\right\rangle=\sum_{\mathbf{i}, \mathbf{j} \in\{0, \ldots, n-1\}^{d}}\left\langle e_{\mathbf{i}}, Q e_{\mathbf{j}}\right\rangle \overline{\left\langle e_{\mathbf{i}}, \log R_{n} e_{\mathbf{j}}\right\rangle} \\
= & \sum_{\mathbf{i}, \mathbf{j} \in\{0, \ldots, n-1\}^{d}}\left\langle e_{\mathbf{i}-\mathbf{j}}, Q e_{\mathbf{0}}\right\rangle \overline{\left\langle e_{\mathbf{i}-\mathbf{j}}, \log R_{n} e_{\mathbf{0}}\right\rangle} \\
= & \sum_{\mathbf{k} \in\{-(n-1), \ldots,+(n-1)\}^{d}}\left(\prod_{i=1}^{d} 2\left(n-\left|k_{i}\right|\right)\right)\left\langle e_{\mathbf{k}}, Q e_{\mathbf{0}}\right\rangle \overline{\left\langle e_{\mathbf{k}}, \log R_{n} e_{\mathbf{0}}\right\rangle} \\
= & (2 n)^{d} \sum_{\mathbf{k} \in\{-(n-1), \ldots,+(n-1)\}^{d}}^{\left\langle e_{\mathbf{k}}, Q e_{\mathbf{0}}\right\rangle \overline{\left\langle e_{\mathbf{k}}, \log R_{n} e_{\mathbf{0}}\right\rangle}} \\
& +2^{d} \sum_{\mathbf{k} \in\{-(n-1), \ldots,(n-1)\}^{d}}^{\left\langle e_{\mathbf{k}}, Q e_{\mathbf{0}}\right\rangle \overline{\left\langle e_{\mathbf{k}}, \log R_{n} e_{\mathbf{0}}\right\rangle}} \\
& \times \sum_{l=0}^{d-1} n^{l}(-1)^{d-l} \sum_{1 \leq i_{1}<\ldots<i_{d-l} \leq d} \prod_{j=1}^{d-l}\left|k_{i_{j}}\right| \\
= & : n^{d} A_{n}+B_{n}
\end{aligned}
$$

where $\mathbf{k}=\left(k_{1}, \ldots, k_{d}\right)$. However, the condition (4.22), as well as the bound $\left|\left\langle e_{\mathbf{k}}, \log R_{n} e_{0}\right\rangle\right| \leq$ $\log (1-\delta)$ imply that

$n\left|\sum_{\mathbf{k} \notin\{-(n-1), \ldots,+(n-1)\}^{d}}\left\langle e_{\mathbf{k}}, Q e_{\mathbf{0}}\right\rangle \overline{\left\langle e_{\mathbf{k}}, \log R_{n} e_{\mathbf{0}}\right\rangle}\right| \leq \log (1-\delta) \sum_{\mathbf{k} \notin\{-(n-1), \ldots,+(n-1)\}^{d}}|\mathbf{k}|\left|\left\langle e_{\mathbf{k}}, Q e_{\mathbf{0}}\right\rangle\right| \rightarrow 0$,

so that

$$
\sum_{\mathbf{k} \in \mathbb{Z}^{d}}\left\langle e_{\mathbf{k}}, Q e_{\mathbf{0}}\right\rangle \overline{\left\langle e_{\mathbf{k}}, \log R_{n} e_{\mathbf{0}}\right\rangle}-\sum_{\mathbf{k} \in\{-(n-1), \ldots,+(n-1)\}^{d}}\left\langle e_{\mathbf{k}}, Q e_{\mathbf{0}}\right\rangle \overline{\left\langle e_{\mathbf{k}}, \log R_{n} e_{\mathbf{0}}\right\rangle}=o\left(n^{-1}\right)
$$

Thus, the term $A_{n}$ in Equation $(4.29)$ has the following asymptotic behavior:

$$
A_{n}=2^{d} \sum_{\mathbf{k} \in \mathbb{Z}^{d}}\left\langle e_{\mathbf{k}}, Q e_{\mathbf{0}}\right\rangle \overline{\left\langle e_{\mathbf{k}}, \log R_{n} e_{\mathbf{0}}\right\rangle}+o\left(n^{-1}\right) .
$$


Similarly

$$
\begin{aligned}
\left|B_{n}\right| & \leq 2^{d} \log (1-\delta) \sum_{l=0}^{d-1} n^{l} \sum_{\mathbf{k} \in \mathbb{Z}^{d}}\left|\left\langle e_{\mathbf{k}}, Q e_{\mathbf{0}}\right\rangle\right| \sum_{1 \leq i_{1}<\ldots<i_{d-l} \leq d} \prod_{j=1}^{d-l}\left|k_{i_{j}}\right| \\
& \leq 2^{d} \log (1-\delta) \sum_{l=0}^{d-1} n^{l} \sum_{\mathbf{k} \in \mathbb{Z}^{d}}|\mathbf{k}|^{d}\left|\left\langle e_{\mathbf{k}}, Q e_{\mathbf{0}}\right\rangle\right|,
\end{aligned}
$$

which implies that $B_{n}=\mathcal{O}\left(n^{d-1}\right)$. Hence, $\operatorname{Tr} Q_{n} \log R_{n}=n^{d} A^{\prime}+\mathcal{O}\left(n^{d-1}\right)$ for some constant $A^{\prime}$. Similarly we prove that the three other terms of Equation (4.18) have the same asymptotic expansion, so that $D\left(\rho_{n} \| \sigma_{n}\right)=n^{d} A^{\prime \prime}+\mathcal{O}\left(n^{d-1}\right)$, for some constant $A^{\prime \prime}$. But from Equation 4.20 (which is a consequence of the multidimensional multivariate Szegö limit theorem (see Lemma 3.1 of [31]), $A^{\prime \prime}=d(\hat{\rho}, \hat{\sigma})$, and Equation 4.23) follows.

Acknowledgements N.D. would like to thank Fernando Brandao, Mark Fannes and Will Matthews for helpful discussions. Y.P. acknowledges the support of ANR contract ANR-14CE25-0003-0, and would like to thank the Statistical Laboratory of the Centre for Mathematical Sciences at University of Cambridge (where part of this work was done) for hospitality. N.D. and C.R. are grateful to Felix Leditzky for help with the figures. All three authors are grateful to Vojkan Jaksic for helpful discussions. They would also like to thank Milan Mosonyi for his valuable comments on an earlier version of the paper.

\section{References}

[1] H. Araki. Relative entropy of states of von Neumann algebras. Publ. Res. Inst. Math. Sci., 11(3):809-833, 1975/76.

[2] H. Araki. Relative entropy for states of von Neumann algebras. II. Publ. Res. Inst. Math. Sci., 13(1):173-192, 1977/78.

[3] S. Attal, A. Joye, and C.-A. Pillet, editors. Open quantum systems. I, volume 1880 of Lecture Notes in Mathematics. Springer-Verlag, Berlin, 2006. The Hamiltonian approach, Lecture notes from the Summer School held in Grenoble, June 16-July 4, 2003.

[4] K. M. R. Audenaert, J. Calsamiglia, R. Muñoz Tapia, E. Bagan, L. Masanes, A. Acin, and F. Verstraete. Discriminating states: The quantum Chernoff bound. Phys. Rev. Lett., 98:160501, April 2007.

[5] R. Bhatia. Matrix analysis, volume 169 of Graduate Texts in Mathematics. SpringerVerlag, New York, 1997.

[6] P. Billingsley. Probability and measure. Wiley Series in Probability and Mathematical Statistics. John Wiley \& Sons, Inc., New York, third edition, 1995. A Wiley-Interscience Publication.

[7] I. Bjelaković, J.-D. Deuschel, T. Krüger, R. Seiler, R. Siegmund-Schultze, and A. Szkoła. Typical Support and Sanov Large Deviations of Correlated States. Communications in Mathematical Physics, 279(2):559-584, February 2008. 
[8] I. Bjelakovic and R. Siegmund-Schultze. An Ergodic Theorem for the Quantum Relative Entropy. Communications in Mathematical Physics, 247(3):697-712, June 2004.

[9] O. Bratteli and D. W. Robinson. Operator algebras and quantum statistical mechanics. 1. Texts and Monographs in Physics. Springer-Verlag, New York, second edition, 1987.

[10] O. Bratteli and D. W. Robinson. Operator algebras and quantum statistical mechanics. 2. Texts and Monographs in Physics. Springer-Verlag, Berlin, second edition, 1997. Equilibrium states. Models in quantum statistical mechanics.

[11] W. Bryc. A remark on the connection between the large deviation principle and the central limit theorem. Statist. Probab. Lett., 18(4):253-256, 1993.

[12] B. Dierckx, M. Fannes, and M. Pogorzelska. Fermionic quasifree states and maps in information theory. Journal of Mathematical Physics, 49(3):032109, September 2008.

[13] W. Feller. An introduction to probability theory and its applications. Vol. II. Second Edition. John Wiley \& Sons, Inc., New York-London-Sydney, 1971.

[14] M. Hayashi. Error exponent in asymmetric quantum hypothesis testing and its application to classical-quantum channel coding. Phys. Rev. A, 76:062301, 2007.

[15] M. Hayashi and V. Y. F. Tan. Asymmetric evaluations of erasure and undetected error probabilities.

[16] M. Hayashi and M. Tomamichel. Correlation detection and an operational interpretation of the Rényi mutual information. (arXiv:1408.6894).

[17] C. W. Helstrom. Quantum detection and estimation theory. J. Statist. Phys., 1:231-252, 1969.

[18] F. Hiai, M. Mosonyi, and M. Hayashi. Quantum hypothesis testing with group symmetry. Journal of Mathematical Physics, 50(10):103304, October 2009.

[19] F. Hiai, M. Mosonyi, and T. Ogawa. Large deviations and Chernoff bound for certain correlated states on a spin chain. Journal of Mathematical Physics, 48(12):123301, June 2007.

[20] F. Hiai, M. Mosonyi, and T. Ogawa. Error exponents in hypothesis testing for correlated states on a spin chain. Journal of Mathematical Physics, 49(3):032112, March 2008.

[21] F. Hiai and D. Petz. The proper formula for relative entropy and its asymptotics in quantum probability. Comm. Math. Phys., 143(1):99-114, 1991.

[22] F. Hiai and D. Petz. Entropy Densities for Algebraic States. Journal of Functional Analysis, 125(1):287-308, October 1994.

[23] A. S. Holevo. An analogue of statistical decision theory and noncommutative probability theory. Trudy Moskov. Mat. Obšč., 26:133-149, 1972.

[24] V. Jakšić, Y. Ogata, C.-A. Pillet, and R. Seiringer. Quantum hypothesis testing and non-equilibrium statistical mechanics. Rev. Math. Phys., 24(6):1230002, 67, 2012. 
[25] V. Jakšić, Y. Ogata, Y. Pautrat, and C.-A. Pillet. Entropic fluctuations in quantum statistical mechanics. an introduction. Quantum Theory from Small to Large Scales, Lecture Notes of the Les Houches Summer School, Volume 95:pp.213-410, 2012.

[26] T. Kato. Perturbation Theory for Linear Operators. Classics in Mathematics. Springer, 1976.

[27] F. Leditzky and N. Datta. Second order asymptotics of mixed quantum source coding via universal codes. (arXiv:1407.6616).

[28] K. Li. Second-order asymptotics for quantum hypothesis testing. Ann. Statist., 42(1):171-189, 2014.

[29] M. Mosonyi. Hypothesis testing for Gaussian states on bosonic lattices. Journal of Mathematical Physics, 50(3).

[30] M. Mosonyi, F. Hiai, T. Ogawa, and M. Fannes. Asymptotic distinguishability measures for shift-invariant quasifree states of fermionic lattice systems. Journal of Mathematical Physics, 49(7):072104, July 2008.

[31] M. Mosonyi, F. Hiai, T. Ogawa, and M. Fannes. Asymptotic distinguishability measures for shift-invariant quasifree states of fermionic lattice systems. J. Math. Phys., 49(7):072104, 11, 2008.

[32] H. Nagaoka. The converse part of the theorem for quantum Hoeffding bound. (arXiv:quant-ph/0611289).

[33] K. Netočný and F. Redig. Large deviations for quantum spin systems. J. Statist. Phys., 117(3-4):521-547, 2004.

[34] M. Nussbaum and A. Szkoła. The Chernoff lower bound for symmetric quantum hypothesis testing. Ann. Statist., 37(2):1040-1057, 2009.

[35] T. Ogawa and M. Hayashi. On error exponents in quantum hypothesis testing. IEEE Trans. Inform. Theory, 50(6):1368-1372, 2004.

[36] T. Ogawa and H. Nagaoka. Strong converse and Stein's lemma in quantum hypothesis testing. IEEE Trans. Inform. Theory, 46(7):2428-2433, 2000.

[37] M. Ohya and D. Petz. Quantum entropy and its use. Texts and Monographs in Physics. Springer-Verlag, Berlin, 1993.

[38] D. Petz. Quasientropies for states of a von Neumann algebra. Publications of the Research Institute for Mathematical Sciences, 21(4):787-800, 1985.

[39] D. Petz. Quasi-entropies for finite quantum systems. Reports on Mathematical Physics, 23(1):57-65, February 1986.

[40] M. Reed and B. Simon. Methods of Modern Mathematical Physics. I. Academic Press, Inc. [Harcourt Brace Jovanovich, Publishers], New York, second edition, 1980. Functional analysis. 
[41] B. Simon. The statistical mechanics of lattice gases. Vol. I. Princeton Series in Physics. Princeton University Press, Princeton, NJ, 1993.

[42] V. Strassen. Asymptotische Abschätzungen in Shannon's Informationstheorie. Trans. Third Prague Conf. Information Theory, pages 689-723, 1962.

[43] V. Y. F. Tan. Asymptotic estimates in information theory with non-vanishing error probabilities. Foundations and Trends® in Communications and Information Theory, 11(1-2):1-184, 2014.

[44] M. Tomamichel and M. Hayashi. A hierarchy of information quantities for finite block length analysis of quantum tasks. IEEE Trans. Inform. Theory, 59(11):7693-7710, 2013.

[45] M. Tomamichel and V. Y. Tan. Second-order asymptotics for the classical capacity of image-additive quantum channels. Communications in Mathematical Physics, 338(1):103-137, 2015.

[46] S. Watanabe and M. Hayashi. Finite-length analysis on tail probability for Markov chain and application to simple hypothesis testing. (arXiv:1401.3801).

[47] M. M. Wilde, J. M. Renes, and S. Guha. Second-order coding rates for pure-loss bosonic channels. Quantum Information Processing, 2015.

\section{A Vitali's theorem and Bryc's theorem}

In this section we state and prove Vitali's theorem, and prove Bryc's theorem (both in the case of one variable).

Theorem 4. Let $r>0$, and let $\left(F_{n}\right)_{n \in \mathbb{N}}$ be a family of analytic functions on $B_{\mathbb{C}}(0, r)$, such that

1. for any $x$ (real) in $(-r,+r), F_{n}(x) \rightarrow_{n \rightarrow \infty} F(x)$,

2. one has the uniform bound $\sup _{n} \sup _{z \in B_{\mathbb{C}}(0, r)}\left|F_{n}(z)\right|<\infty$,

then

1. the function $F$ so defined can be extended to an analytic function on $B_{\mathbb{C}}(0, r)$,

2. for any $k \in\{0\} \cup \mathbb{N}$, the $k$-th derivative $F_{n}^{(k)}$ converges uniformly on any compact subset of $B_{\mathbb{C}}(0, r)$ to the $k$-th derivative $F^{(k)}$.

Proof. For any arbitrary $r_{0} \in(0, r)$, let $\Gamma_{r_{0}}$ denote the circle centered at the origin with radius $r_{0}$. We have for any $n$ and any $z$ in $B_{\mathbb{C}}\left(0, r_{0}\right)$

$$
F_{n}^{(k)}(z)=\frac{k !}{2 \mathrm{i} \pi} \int_{\Gamma_{r_{0}}} \frac{F_{n}(u)}{(u-z)^{k+1}} \mathrm{~d} u .
$$

Assumption 2 implies that the family of derivatives $\left(F_{n}^{\prime}\right)_{n \in \mathbb{N}}$ is uniformly bounded on the closed ball $\overline{B_{\mathbb{C}}\left(0, r_{0}\right)}$. Since this holds for any arbitrary $r_{0} \in(0, r),\left(F_{n}\right)_{n \in \mathbb{N}}$ is equicontinuous 
on any compact subset of $B_{\mathbb{C}}(0, r)$. The Arzela-Ascoli theorem implies that there exists a subsequence $\left(F_{\varphi(n)}\right)_{n}$ of $\left(F_{n}\right)_{n}$ which converges uniformly to an analytic limit $G$ on $\overline{B_{\mathbb{C}}\left(0, r_{0}\right)}$. Necessarily, relation A.1) holds for $G$, which is therefore analytic on $B_{\mathbb{C}}(0, r)$. By assumption 1 , on the interval $(-r, r), G$ equals $F$ necessarily. This, uniquely determines $G$ on $B_{\mathbb{C}}(0, r)$, so that $\left(F_{n}\right)_{n}$ itself converges to an analytic function which is an extension of $F$. The convergence of derivatives follows from Equation A.1.

We now turn to a proof of Bryc's theorem, stated as Theorem 2.

Proof. The analyticity of $H_{n}$ in a neighbourhood of zero shows that the random variables $X_{n}$ have moments of all orders. By a direct application of Vitali's theorem, the function $H$ is analytic on $B_{\mathbb{C}}(0, r)$ and $\frac{1}{w_{n}} H_{n}$ converges uniformly on any compact subset of $B_{\mathbb{C}}(0, r)$ to $H$. Replacing $X_{n}$ with $X_{n}+H_{n}^{\prime}(0)$ we can assume that $H_{n}^{\prime}(0)=0$ for all $n$. For $R>0$ denote by $\Gamma_{R}$ the circle centered at the origin, with radius $R$. Cauchy's formula gives, for any $0<r_{0}<r$, and $k \in \mathbb{N}$

$$
\begin{aligned}
\frac{\mathrm{d}^{k}}{\mathrm{~d} z^{k}} H(z) & =\frac{k !}{2 \mathrm{i} \pi} \int_{\Gamma_{r_{0} / \sqrt{w_{n}}}} \frac{H(u)}{u^{k+1}} \mathrm{~d} u \\
& =\lim _{n \rightarrow \infty} \frac{k !}{w_{n} 2 \mathrm{i} \pi} \int_{\Gamma_{r_{0} / \sqrt{w_{n}}}} \frac{H_{n}(u)}{u^{k+1}} \mathrm{~d} u
\end{aligned}
$$

and a simple change of variable $u=u^{\prime} / \sqrt{w_{n}}$ gives

$$
H^{(k)}(0)=\lim _{n \rightarrow \infty} w_{n}^{k / 2-1} \frac{k !}{2 \mathrm{i} \pi} \int_{\Gamma_{r_{0}}} \frac{H_{n}\left(u / \sqrt{w_{n}}\right)}{u^{k+1}} \mathrm{~d} u .
$$

However,

$$
\frac{k !}{2 \mathrm{i} \pi} \int_{\Gamma_{r_{0}}} \frac{H_{n}\left(u / \sqrt{w_{n}}\right)}{u^{k+1}} \mathrm{~d} u=\left.\frac{\mathrm{d}^{k}}{\mathrm{~d} z^{k}}\right|_{z=0} \log \mathbb{E}\left(\exp -z \frac{X_{n}}{\sqrt{w_{n}}}\right),
$$

so that

$$
H^{(k)}(0)=\left.\lim _{n \rightarrow \infty} w_{n}^{k / 2-1} \frac{\mathrm{d}^{k}}{\mathrm{~d} z^{k}}\right|_{z=0} \log \mathbb{E}\left(\exp -z \frac{X_{n}}{\sqrt{w_{n}}}\right) .
$$

Therefore, for $k \geq 3$,

$$
\left.\lim _{n \rightarrow \infty} \frac{\mathrm{d}^{k}}{\mathrm{~d} z^{k}}\right|_{z=0} \log \mathbb{E}\left(\exp -z \frac{X_{n}}{\sqrt{w_{n}}}\right)=0
$$

whereas for $k=2$

$$
\left.\lim _{n \rightarrow \infty} \frac{\mathrm{d}^{2}}{\mathrm{~d} z^{2}}\right|_{z=0} \log \mathbb{E}\left(\exp -z \frac{X_{n}}{\sqrt{w_{n}}}\right)=\lim _{n \rightarrow \infty} \frac{1}{w_{n}} H_{n}^{\prime \prime}(0)=H^{\prime \prime}(0)
$$

and by the choice that $H_{n}^{\prime}(0)=0$ for any $n$,

$$
\left.\lim _{n \rightarrow \infty} \frac{\mathrm{d}}{\mathrm{d} z}\right|_{z=0} \log \mathbb{E}\left(\exp -z \frac{X_{n}}{\sqrt{w_{n}}}\right)=\lim _{n \rightarrow \infty} \frac{1}{\sqrt{w_{n}}} H_{n}^{\prime}(0)=0 .
$$

Therefore, the cumulant of order $k$ of $\left(X_{n} / \sqrt{w_{n}}\right)_{n \in \mathbb{N}}$ converges to the cumulant of order $k$ of the centered normal distribution $\mathcal{N}\left(0, H^{\prime \prime}(0)\right)$, so that the same is true of moments. Since the normal distribution is determined by its moments, the sequence $\left(X_{n} / \sqrt{w_{n}}\right)_{n \in \mathbb{N}}$ converges in law to $\mathcal{N}\left(0, H^{\prime \prime}(0)\right)$ (see Theorem 30.2 and Example 30.1 in [6]). 


\section{B Proof of Lemma 2 and Corollary 2}

Proof of Lemma 2 We first prove the upper bound in Equation (3.11). In order to do so, we use the following inequality (see 24]): For any $A, B \in \mathcal{P}_{\mathbb{R}_{+}}(\mathcal{H})$ and $s$ in $[0,1]$,

$$
\frac{1}{2}(\operatorname{Tr} A+\operatorname{Tr} B-\operatorname{Tr}|A-B|) \leq \operatorname{Tr} A^{1-s} B^{s} .
$$

For a given test $T$,

$$
\begin{aligned}
\mathrm{e}_{\text {sym }}(A, B, T) & =\operatorname{Tr}(A(\mathbb{I}-T))+\operatorname{Tr}(B T) \\
& =\operatorname{Tr} A+\operatorname{Tr}(T(B-A)) .
\end{aligned}
$$

Therefore, by Equation (3.10),

$$
\begin{aligned}
\mathrm{e}_{\mathrm{sym}}^{*}(A, B) & =\operatorname{Tr} A-\operatorname{Tr}(A-B) P_{+}(A-B) \\
& =1 / 2(\operatorname{Tr} A+\operatorname{Tr} B-\operatorname{Tr}|A-B|),
\end{aligned}
$$

where $P_{\mathbb{R}_{+}}(A-B)$ denotes the projection onto the support of $(A-B)_{+}$. From Equation $(2.10)$ we have that $\left\langle\Omega_{B}, \Delta_{A \mid B}^{s} \Omega_{B}\right\rangle=\operatorname{Tr}\left(A^{s} B^{1-s}\right)$ where $\Omega_{B}=B^{1 / 2}$. Hence using Equation (B.1) we obtain

$$
\left\langle\Omega_{B}, \Delta_{A \mid B}^{s} \Omega_{B}\right\rangle \geq 1 / 2(\operatorname{Tr} A+\operatorname{Tr} B-\operatorname{Tr}|A-B|)=\mathrm{e}_{\mathrm{sym}}^{*}(A, B) .
$$

Next we prove the lower bound in Equation (3.11). By Equation (3.10) we know that the infimum in Equation (3.9) of $e_{\mathrm{sym}}^{*}$ is attained for a test given by an orthogonal projection which we denote by $P$. For such a test, $P$, using spectral decompositions of $A$ and $B$ we can write

$$
\begin{aligned}
\operatorname{Tr}(A(\mathbb{I}-P)) & =\sum_{\lambda \in \operatorname{sp}(A)} \lambda \operatorname{Tr}\left((\mathbb{I}-P) P_{\lambda}(A)(\mathbb{I}-P)\right) \\
& =\sum_{(\lambda, \mu) \in \operatorname{sp}(\rho) \times \operatorname{sp}(B)} \lambda \mu^{-1} \operatorname{Tr}\left(B(\mathbb{I}-P) P_{\lambda}(A)(\mathbb{I}-P) P_{\mu}(B)\right) .
\end{aligned}
$$

Similarly,

$$
\operatorname{Tr}(B P)=\sum_{(\lambda, \mu) \in \operatorname{sp}(A) \times \operatorname{sp}(B)} \operatorname{Tr}\left(B P P_{\lambda}(A) P P_{\mu}(B)\right)
$$

where we have used the fact that $\sum_{\lambda} P_{\lambda}(A)=\mathbb{I}=\sum_{\mu} P_{\mu}(B)$ since $A, B \in \mathcal{P}_{\mathbb{R}_{+}}(\mathcal{H})$. Therefore as for any $\kappa \geq 0$, denoting $\bar{P}=\mathbb{I}-P$, we have

$$
\begin{aligned}
\kappa \bar{P} P_{\lambda}(A) \bar{P}+P P_{\lambda}(A) P & =\frac{\kappa}{1+\kappa} P_{\lambda}(A)+\frac{1}{1+\kappa}(1-(1+\kappa) \bar{P}) P_{\lambda}(A)(1-(1+\kappa) \bar{P}) \\
& \geq \frac{\kappa}{1+\kappa} P_{\lambda}(A)
\end{aligned}
$$


and hence we obtain

$$
\begin{aligned}
\operatorname{Tr}(B P)+\operatorname{Tr}(A(\mathbb{I}-P)) & \geq \sum_{(\lambda, \mu) \in \operatorname{sp}(A) \times \operatorname{sp}(B)} \frac{\lambda / \mu}{1+\lambda / \mu} \operatorname{Tr}\left(B P_{\lambda}(A) P_{\mu}(B)\right) \\
& =\left\langle\Omega_{B}, \Delta_{A \mid B}\left(1+\Delta_{A \mid B}\right)^{-1} \Omega_{B}\right\rangle
\end{aligned}
$$

and the lower bound in Equation (3.11) follows.

Proof of Corollary 2 Lemma 2 implies that for any $\theta \in \mathbb{R}$,

$$
\begin{aligned}
\mathrm{e}_{\mathrm{sym}}^{*}\left(\sigma, \mathrm{e}^{-\theta} \rho\right) & \geq\left\langle\Omega_{\mathrm{e}^{-\theta} \rho}, \Delta_{\sigma \mid \mathrm{e}^{-\theta} \rho}\left(1+\Delta_{\sigma \mid \mathrm{e}^{-\theta} \rho}\right)^{-1} \Omega_{\mathrm{e}^{-\theta} \rho}\right\rangle \\
& =\mathrm{e}^{-\theta}\left\langle\Omega_{\rho}, \mathrm{e}^{\theta} \Delta_{\sigma \mid \rho}\left(1+\mathrm{e}^{\theta} \Delta_{\sigma \mid \rho}\right)^{-1} \Omega_{\rho}\right\rangle \\
& =\int_{\mathbb{R}} \frac{\mathrm{e}^{-\theta}}{1+\mathrm{e}^{x-\theta}} d \mu_{\sigma \mid \rho}(x)
\end{aligned}
$$

and the result follows from Markov's inequality.

\section{Proof of Proposition 1}

Let the spectral decompositions of the states $\rho$ and $\sigma$ be given as follows,

$$
\rho=\sum_{\lambda \in \operatorname{sp}(\rho)} \lambda P_{\lambda}(\rho) \quad \sigma=\sum_{\mu \in \operatorname{sp}(\sigma)} \mu P_{\mu}(\sigma)
$$

Denote for any $\lambda \in \operatorname{sp}(\rho)$ :

$$
Q_{\lambda}:=P_{(-\infty, \lambda / L]}(\sigma)=\sum_{\mu \in \operatorname{sp}(\sigma): L \mu \leq \lambda} P_{\mu}(\sigma) .
$$

$Q_{\lambda}$ then increases with $\lambda$. The operator

$$
\tilde{T}:=\sum_{\lambda \in \operatorname{sp}(\rho), \mu \in \operatorname{sp}(\sigma): L \mu \leq \lambda} P_{\mu}(\sigma) P_{\lambda}(\rho) P_{\mu}(\sigma)
$$

is well-defined and nonnegative. We define our test $T$ by the projection onto the support of $\tilde{T}$. Now fix $\lambda \in \operatorname{sp}(\rho)$. For any $\varphi \in \operatorname{ran}\left(P_{\lambda}(\rho)\right)$ of unit norm, one has $P_{\lambda}(\rho) \geq|\varphi\rangle\langle\varphi|$, where $|\varphi\rangle\langle\varphi|$ denotes the projector onto $\operatorname{span}(\varphi)$, and therefore for any $\mu \in \operatorname{sp}(\sigma)$ such that $L \mu \leq \lambda$ one has $\tilde{T} \geq\left|P_{\mu}(\sigma) \varphi\right\rangle\left\langle P_{\mu}(\sigma) \varphi\right|$. This implies that $P_{\mu}(\sigma) \varphi \in \operatorname{supp}(\tilde{T})$. Hence

$$
T \geq \frac{\left|P_{\mu}(\sigma) \varphi\right\rangle\left\langle P_{\mu}(\sigma) \varphi\right|}{\left\|P_{\mu}(\sigma) \varphi\right\|^{2}}
$$

Since $\left\{P_{\mu}(\sigma) \varphi\right\}_{\mu \in \operatorname{sp}(\sigma)}$ form a family of orthogonal vectors, the following inequality holds,

$$
T \geq \sum_{\mu \in \operatorname{sp}(\sigma): L \mu \leq \lambda} \frac{\left|P_{\mu}(\sigma) \varphi\right\rangle\left\langle P_{\mu}(\sigma) \varphi\right|}{\left\|P_{\mu}(\sigma) \varphi\right\|^{2}} .
$$


Moreover, since

$$
\operatorname{Tr}\left(|\varphi\rangle\left\langle\varphi|| P_{\mu}(\sigma) \varphi\right\rangle\left\langle P_{\mu}(\sigma) \varphi\right|\right)=\left\|P_{\mu}(\sigma) \varphi\right\|^{4}
$$

we have that

$$
\operatorname{Tr}(|\varphi\rangle\langle\varphi| T) \geq \sum_{\mu \in \operatorname{sp}(\sigma): L \mu \leq \lambda}\left\|P_{\mu}(\sigma) \varphi\right\|^{2}=\sum_{\mu \in \operatorname{sp}(\sigma): L \mu \leq \lambda} \operatorname{Tr}\left(|\varphi\rangle\langle\varphi| P_{\mu}(\sigma)\right)
$$

for $\varphi \in \operatorname{ran}\left(P_{\lambda}(\rho)\right)$. Thus by writing $P_{\lambda}(\rho)=\sum_{i=1}^{\operatorname{dim}\left(\operatorname{ran}\left(P_{\lambda}(\rho)\right)\right)}\left|\varphi_{i}\right\rangle\left\langle\varphi_{i}\right|$, where $\left\{\varphi_{i}\right\}_{i}$ is an orthonormal basis for $\operatorname{ran}\left(P_{\lambda}(\rho)\right)$,

$$
\operatorname{Tr} P_{\lambda}(\rho) T \geq \sum_{\mu \in \operatorname{sp}(\sigma): L \mu \leq \lambda} \operatorname{Tr} P_{\lambda}(\rho) P_{\mu}(\sigma)
$$

and

$$
\begin{aligned}
\operatorname{Tr} \rho T \geq \sum_{\lambda \in \operatorname{sp}(\rho), \mu \in \operatorname{sp}(\sigma): L \mu \leq \lambda} \lambda \operatorname{Tr} P_{\lambda}(\rho) P_{\mu}(\sigma) & =\sum_{\lambda \in \operatorname{sp}(\rho), \mu \in \operatorname{sp}(\sigma): L \mu \leq \lambda} \operatorname{Tr}\left(\rho^{1 / 2} P_{\lambda}(\rho) \rho^{1 / 2} P_{\mu}(\sigma)\right) \\
& =\left\langle\Omega_{\rho}, P_{[L,+\infty)}\left(\Delta_{\rho \mid \sigma}\right) \Omega_{\rho}\right\rangle .
\end{aligned}
$$

Since $\Delta_{\rho \mid \sigma}$ is a positive operator, this proves the first inequality in (3.14).

To prove the second inequality in (3.14), first observe that for any $\lambda \in \operatorname{sp}(\rho), \mu \in \operatorname{sp}(\sigma)$ :

$$
\operatorname{ran}\left(P_{\mu}(\sigma) P_{\lambda}(\rho)\right)=\operatorname{supp}\left(P_{\mu}(\sigma) P_{\lambda}(\rho) P_{\mu}(\sigma)\right) .
$$

Indeed, using the decomposition $P_{\lambda}(\rho)=\sum_{i=1}^{\operatorname{dim}\left(\operatorname{ran}\left(P_{\lambda}(\rho)\right)\right)}\left|\varphi_{i}\right\rangle\left\langle\varphi_{i}\right|$, where $\left\{\varphi_{i}\right\}$ is an orthonormal basis for $\operatorname{ran}\left(P_{\lambda}(\rho)\right)$, we obtain

$$
P_{\mu}(\sigma) P_{\lambda}(\rho) P_{\mu}(\sigma)=\sum_{i=1}^{\operatorname{dim} \operatorname{ran}\left(P_{\lambda}(\rho)\right)}\left|P_{\mu}(\sigma) \varphi_{i}\right\rangle\left\langle P_{\mu}(\sigma) \varphi_{i}\right|
$$

which implies that the support of $P_{\mu}(\sigma) P_{\lambda}(\rho) P_{\mu}(\sigma)$ is the span of the set of vectors $\left\{P_{\mu}(\sigma) \varphi_{i}\right\}_{i}$, and is hence the range of $P_{\mu}(\sigma) P_{\lambda}(\rho)$. Then denote for $\lambda \in \operatorname{sp}(\rho)$,

$$
\tilde{\mathcal{A}}_{\lambda}=\bigvee_{\mu \in \operatorname{sp}(\sigma): \lambda / \mu \geq L} \operatorname{ran}\left(P_{\mu}(\sigma) P_{\lambda}(\rho)\right)=\bigvee_{\mu \in \operatorname{sp}(\sigma): \lambda / \mu \geq L} \operatorname{supp}\left(P_{\mu}(\sigma) P_{\lambda}(\rho) P_{\mu}(\sigma)\right)
$$

To complete the proof we employ a Gram-Schmidt orthogonalization procedure as in [28]. We first order the eigenvalues of $\rho$ and denote them as $\lambda_{1}, \lambda_{2}, \ldots, \lambda_{d_{\rho}}$ where $d_{\rho}$ is the number of different eigenvalues of $\rho$, and $\lambda_{1}>\lambda_{2}>\ldots>\lambda_{d_{\rho}}$. Define a family $\left(\mathcal{A}_{i}\right)_{1 \leq i \leq d_{\rho}}$ of subspaces of $\mathcal{H}$ by $\mathcal{A}_{1}:=\tilde{\mathcal{A}}_{\lambda_{1}}$ and, $\forall 1 \leq k \leq d_{\rho}-1$,

$$
\mathcal{A}_{k+1}:=\left(\tilde{\mathcal{A}}_{\lambda_{1}}+\ldots+\tilde{\mathcal{A}}_{\lambda_{k+1}}\right) \cap\left(\tilde{\mathcal{A}}_{\lambda_{1}}+\ldots+\tilde{\mathcal{A}}_{\lambda_{k}}\right)^{\perp} .
$$

The subspaces $\mathcal{A}_{k}$ are mutually orthogonal by construction. Moreover, it can be shown by induction that for any $k$,

$$
\mathcal{A}_{1}+\ldots+\mathcal{A}_{k}=\tilde{\mathcal{A}}_{\lambda_{1}}+\ldots+\tilde{\mathcal{A}}_{\lambda_{k}}
$$


By definition, $T$ is the projection on

$$
\begin{aligned}
\bigvee_{\lambda \in \operatorname{sp}(\rho), \mu \in \operatorname{sp}(\sigma): \lambda \geq L \mu} \operatorname{supp}\left(P_{\mu}(\sigma) P_{\lambda}(\rho) P_{\mu}(\sigma)\right) & =\bigvee_{\lambda \in \operatorname{sp}(\rho), \mu \in \operatorname{sp}(\sigma): \lambda \geq L \mu} \operatorname{ran}\left(P_{\mu}(\sigma) P_{\lambda}(\rho)\right) \\
& =\bigvee_{\lambda} \tilde{\mathcal{A}}_{\lambda}=\bigoplus_{k=1}^{d_{\rho}} \mathcal{A}_{k}
\end{aligned}
$$

so that $T=\sum_{k}^{d_{\rho}} P_{\mathcal{A}_{k}}$ where, for each $k, P_{\mathcal{A}_{k}}$ is the orthogonal projector onto $\mathcal{A}_{k}$. Note that

$$
\mathcal{A}_{k} \subset \tilde{\mathcal{A}}_{\lambda_{k}} \Rightarrow \operatorname{dim} \mathcal{A}_{k} \leq \operatorname{dim} \tilde{\mathcal{A}}_{\lambda_{k}}
$$

Using the definition of $\tilde{\mathcal{A}}_{\lambda_{k}}$, one finally gets

$$
\operatorname{Tr} P_{\mathcal{A}_{k}}=\operatorname{dim} \mathcal{A}_{k} \leq \operatorname{dim} \tilde{\mathcal{A}}_{\lambda_{k}} \leq \operatorname{Tr} P_{\lambda_{k}}(\rho) .
$$

We then obtain

$$
\begin{aligned}
\operatorname{Tr} \sigma T & =\sum_{\mu \in \operatorname{sp}(\sigma)} \sum_{k=1}^{d_{\rho}} \mu \operatorname{Tr}\left(P_{\mu}(\sigma) P_{\mathcal{A}_{k}}\right) \\
& =\sum_{k=1}^{d_{\rho}} \sum_{\substack{\mu \in \operatorname{sp}(\sigma): \\
\lambda_{k} / \mu \geq L}} \mu \operatorname{Tr}\left(P_{\mu}(\sigma) P_{\mathcal{A}_{k}}\right) \\
& \leq L^{-1} \sum_{\substack{\mu \in \operatorname{sp}(\sigma): \\
\lambda_{k} / \mu \geq L}} \sum_{k=1}^{d_{\rho}} \lambda_{k} \operatorname{Tr}\left(P_{\mu}(\sigma) P_{\mathcal{A}_{k}}\right) \\
& \leq L^{-1} \sum_{\substack{\mu \in \operatorname{sp}(\sigma) \\
d_{\rho}}}^{d_{\rho}} \lambda_{k} \operatorname{Tr}\left(P_{\mu}(\sigma) P_{\mathcal{A}_{k}}\right)=L^{-1} \sum_{k=1}^{d_{\rho}} \lambda_{k} \operatorname{Tr}\left(P_{\mathcal{A}_{k}}\right) \\
& \leq L^{-1} \sum_{k=1}^{\lambda_{k}} \operatorname{Tr}\left(P_{\lambda_{k}}(\rho)\right)=L^{-1} \operatorname{Tr}(\rho)=L^{-1},
\end{aligned}
$$

where the second line follows from the fact that $P_{\mu}(\sigma) P_{\mathcal{A}_{k}}=0$ unless $\lambda_{k} / \mu \geq L$ (by C.2 and (C.6) $)$, and the last one from (C.7). This concludes the proof. 